\title{
UniT: Unified Knowledge Transfer for Any-shot Object Detection and Segmentation
}

\author{
Siddhesh Khandelwal ${ }^{*, 1,2} \quad$ Raghav Goyal $^{*, 1,2} \quad$ Leonid Sigal $^{1,2,3}$ \\ ${ }^{1}$ Department of Computer Science, University of British Columbia \\ Vancouver, BC, Canada \\ ${ }^{2}$ Vector Institute for AI \\ ${ }^{3}$ CIFAR AI Chair \\ skhandel@cs.ubc.ca rgoyall4@cs.ubc.ca lsigal@cs.ubc.ca
}

\begin{abstract}
Methods for object detection and segmentation rely on large scale instance-level annotations for training, which are difficult and time-consuming to collect. Efforts to alleviate this look at varying degrees and quality of supervision. Weakly-supervised approaches draw on image-level labels to build detectors/segmentors, while zero/few-shot methods assume abundant instance-level data for a set of base classes, and none to a few examples for novel classes. This taxonomy has largely siloed algorithmic designs. In this work, we aim to bridge this divide by proposing an intuitive and unified semi-supervised model that is applicable to a range of supervision: from zero to a few instance-level samples per novel class. For base classes, our model learns a mapping from weakly-supervised to fully-supervised detectors/segmentors. By learning and leveraging visual and lingual similarities between the novel and base classes, we transfer those mappings to obtain detectors/segmentors for novel classes; refining them with a few novel class instance-level annotated samples, if available. The overall model is end-to-end trainable and highly flexible. Through extensive experiments on MSCOCO [32] and Pascal VOC [14] benchmark datasets we show improved performance in a variety of settings.
\end{abstract}

\section{Introduction}

Over the past decade CNNs have emerged as the dominant building blocks for various computer vision understanding tasks, including object classification [21, 44, 51], detection [33, 41, 42], and segmentation [8, 20]. Architectures based on Faster R-CNN [42], Mask R-CNN [20] and YOLO [41] have achieved impressive performance on a variety of core vision tasks. However, traditional CNN-based approaches rely on lots of supervised data for which the annotation efforts can be time-consuming and expensive [22, 29]. While imagelevel class labels are easy to obtain, more structured labels

\footnotetext{
*Denotes equal contribution
}

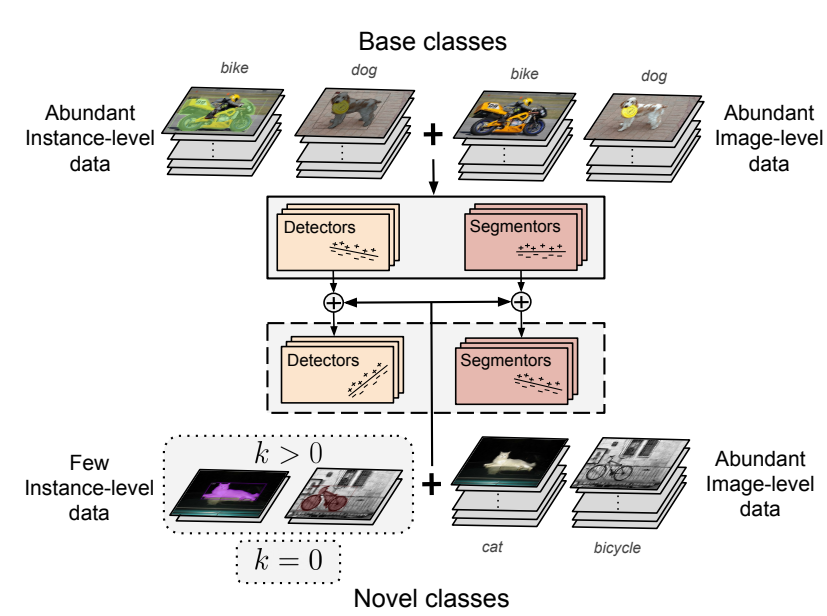

Figure 1: Semi-supervised Any-shot Detection and Segmentation. The data used in our setting is categorized in two ways: (1) image-level classification data for all the object classes, and (2) abundant instance data for a set of base object classes and limited (possibly zero) instance data for a set of novel object classes, with the aim to obtain a model that learns to detect/segment both base and novel objects at test time.

such as bounding boxes or segmentations are difficult and expensive ${ }^{1}$. Further, in certain domains (e.g., medical imaging) more detailed labels may require subject expertise. The growing need for efficient learning has motivated development of various approaches and research sub-communities.

On one end of the spectrum, zero-shot learning methods require no visual data and use auxiliary information, such as attributes or class names, to form detectors for unseen classes from related seen category detectors [3, 16, 39, 64]. Weakly-supervised learning methods [2, 5, 12, 29, 60] aim to utilize readily available coarse image-level labels for more granular downstream tasks, such as object detection [3,39]

\footnotetext{
${ }^{1}$ Segmentation annotations in PASCAL VOC take 239.7 seconds/image, on average, as compared to 20 seconds/image for image-level labels [4].
} 
and segmentation [29, 70]. Most recently, few-shot learning $[1,40,48,59]$ has emerged as a learning-to-learn paradigm which either learns from few labels directly or by simulation of few-shot learning paradigm through meta-learning $[15,46,56]$. An interesting class of semi-supervised methods $[17,22,26,55,57,67]$ have emerged which aim to transfer knowledge from abundant base classes to data-starved novel classes, especially for granular instance-level visual understanding tasks. However, to date, there isn't a single, unified framework that can effectively leverage various forms and amounts of training data (zero-shot to fully supervised).

We make two fundamental observations that motivate our work. First, image-level supervision is abundant, while instance-level structured labels, such as bounding boxes and segmentation masks, are expensive and scarce. This is reflected in the scales of widely used datasets where classification tasks have $>5 \mathrm{~K}$ classes $[28,51]$ while the popular object detection/segmentation datasets, like MSCOCO [32], have annotations for only 80 classes. A similar observation was initially made by Hoffman et al. [22] and other semisupervised [26, 55, 57] approaches. Second, the assumption of no instance-level supervision for target classes (as is the case for semi-supervised [22, 26, 55, 57] and zero-shot methods $[3,16,39,64])$ is artificial. In practice, it is often easy to collect few instance-level annotations and, in general, a good object detection/segmentation model should be robust and work with any amount of available instance-level supervision. Our motivation is to bridge weakly-supervised, zero- and few-shot learning paradigms to build an expressive, simple, and interpretable model that can operate across types (weak/strong) and amounts of instance-level supervision (from 0 to $90+$ instance-level samples per class).

In this work, we develop a unified semi-supervised framework (UniT) for object detection and segmentation that scales with different levels of instance-level supervision ranging from no-data, to a few, to fully supervised (see Figure 1). The data used in our problem is categorized in two ways, (1) image-level classification data for all the object classes, and (2) abundant detection data for a set of base object classes and limited (possibly zero) detection data for a set of novel object classes, with the aim to obtain a model that learns to detect both base and novel objects at test time.

Our algorithm, illustrated in Figure 2, jointly learns weakdetectors for all the object classes, from image-level classification data, and supervised regressors/segmentors on top of those for base classes (based on instance-level annotations in a supervised manner). The classifiers, regressors and segmentors of the novel classes are expressed as a weighted linear combination of its base class counterparts. The weights of the combination are determined by a multimodal similarity measure: lingual and visual. The key insight of our approach is to utilize the multi-modal similarity measure between the novel and base classes to enable effec- tive knowledge transfer and adaptation. The adopted novel classifier/regressors/segmentors can further be refined based on instance-level supervision, if any available. We experiment with the widely-used detection/segmentation datasets - Pascal VOC [13] and MSCOCO [32], and compare our method with state-of-the-art few-shot, weakly-supervised, and semi-supervised object detection/segmentation methods.

Contributions: Our contributions can be summarized as follows: (1) We study the problem of semi-supervised object detection and segmentation in light of image-level supervision and limited instance-level annotations, ranging from no data (zero-shot) to a few (few-shot); (2) We propose a general, unified, interpretable and flexible end-to-end framework that can adopt classifiers/detectors/segmentors for novel classes by expressing them as linear combinations of their base class counterparts. In doing so, we leverage a learned multimodal (lingual + visual) similarity metric. (3) In the context of our model, we explore the relative importance of weak image-level supervision, compared to strong instance-level supervision, and find that under a small fixed annotation budget, image-level supervision is more important. (4) We illustrate flexibility and effectiveness of our model by applying it to a variety of tasks (object detection and segmentation) and datasets (Pascal VOC [13], MSCOCO [32]); showing state-of-the-art performance. We get up to $23 \%$ relative improvement in mAP over the closest semi-supervised methods [17], and as much as $16 \%$ improvement with respect the best performing few-shot method [61] under the same fixed annotation budget. We conduct comprehensive comparisons across settings, tasks, types and levels of supervision.

\section{Related Work}

Few-shot object detection: Object detection with limited data was initially explored in a transfer learning setting by Chen et al. [7]. In the context of meta-learning [1, 15, 40, 48, 59], Kang et al. [24] developed a few-shot model where the learning procedure is divided into two phases: first the model is trained on a set of base classes with abundant data using episodic tasks, then, in the second phase, a few examples of novel classes and base classes are used for fine tuning the model. Following this formulation, $[62,66]$ employed better performing architecture - Faster R-CNN [42], instead of a one-stage YOLOv2 [41]. Yan et al. [66] extended the problem formulation to account for segmentation in addition to detection. In contrast to the above approaches, Wang et al. [61] showed that meta-learning is not a crucial ingredient to Few-shot object detection, and simple fine-tuning produces better detectors. Similar to the above works, we also adopt the two-phase learning procedure. However, we fundamentally differ in assuming that easily attainable extra supervision, in the form of image-level data, over all the classes is available. Unlike [62], we learn a semantic mapping between weakly-supervised detectors and 
detectors obtained using a large number of examples.

Weakly-supervised object detection: Weak supervision in object detection takes the form of image-level labels, usually coupled with bounding box proposals [58, 72], thereby representing each image as a bag of instances [2, 5, 9, 12, 18, 43, 49, 53, 54, 60, 69]. Bilen et al. [5] proposed an end-to-end architecture which softly labeled object proposals and uses a detection stream, in addition to classification stream, to classify them. Further extensions followed, Diba et al. [12] incorporated better proposals into a cascaded deep network; Tang et al. [54] proposed an Online Instance Classifier Refinement (OICR) algorithm which iteratively refines predictions. More recently, further improvements were made by combining weakly-supervised learning with strongly-supervised detectors, by treating predicted locations from the weakly-supervised detector as pseudo-labels for a strongly-supervised variant $[2,60]$. In this work, we choose to adopt and build on top of single-stage OICR [54], hence enabling end-to-end training. However, our approach is not limited to the choice of weakly-supervised architecture.

Semi-supervised object detection: Approaches under semi-supervised setup assume abundant detection data for base classes and no detection data for novel classes, in addition to weak supervision for all the classes. The methods in this category first learn weak classifiers for all classes using abundant weak supervision, then fine-tune base classifiers into detectors using abundant detection data, and finally transfer this transformation to obtain detectors for novel classes using an external (or learned) similarity measure between base and novel classes. LSDA [22], being the first, formed similarity based on L2-normalized weak classifier weights. Tang et al. [55] extended this approach to include semantic and visual similarity explicitly. DOCK [26] expanded the types of similarities to include spatial and attribute cues using external knowledge sources. Other works leverage semantic hierarchies of classes, such as Yang et al. [67] proposes a class split based on granularity of classes, and transfers knowledge from coarse to fine grained classes. Uijlings et al. [57] uses a proposal generator trained on base classes, and transfers the proposals from base to novel classes by computing their similarity on a tree based on Imagenet semantic hierarchy [44]. Similar to the above methods we also use visual and lingual similarities between base and novel classes, but consider a more general problem setting where we have varying degrees of detection supervision for novel classes ranging from zero to a few $k$-samples per class.

Unique, and closest to our setup, is NOTE-RCNN [17]. In [17], few- $k$ detection samples for novel classes are used as seed annotations, based on which training-mining [54, 57] is employed. Specifically, they initialize detectors for novel classes by training them with few seed annotations, and iteratively refine them by retraining with mined bounding boxes for novel classes. They transfer knowledge indirectly in the form of losses that act as regularizers. Our approach, on the other hand, takes on a simpler and more intuitive direction where we first transfer the mappings from base to novel classes, and use few seed annotations (if available) to fine-tune the detectors. Despite being simpler, our approach is more accurate, and works in the $k=0$ regime. Further, unlike all the above semi-supervised approaches, we transfer across tasks, including regression and segmentation.

Zero-shot object detection: Zero-shot approaches rely on auxiliary semantic information to connect base and novel classes; e.g., text description of object labels or their attributes [3, 16, 39, 64]. A common strategy is to represent all classes as prototypes in the semantic embedding space and to learn a mapping from visual features to this embedding space using base class data; classification is then obtained using nearest distance to novel prototypes. This approach was expended to detection in [10, 27, 30, 45, 68, 71]. Bansal et al. [3], similarly, proposed method to deal with situations where objects from novel/unseen classes are present in the background regions. We too explore the setting where we are not provided with any instance data for novel classes, but in addition assume weak-supervision for novel object classes in the form of readily available [28] image-level annotations.

\section{Problem Formulation}

Here we formally introduce the semi-supervised any-shot object detection / segmentation setup. We start by assuming image-level supervision for all the classes denoted by $\mathcal{D}^{\text {class }}=\left\{\left(\mathbf{x}_{i}, \mathbf{a}_{i}\right)\right\}$, where each image $\mathbf{x}_{i}$ is annotated with a label $\mathbf{a}_{i} \in\{0,1\}^{|\mathcal{C}|}$, where $a_{i}^{j}=1$ if image $\mathbf{x}_{i}$ contains at least one $j$-th object, indicating its presence; $\mathbf{a}_{i}=\left\{a_{i}^{j}\right\}_{j=1}^{|\mathcal{C}|}$ with $|\mathcal{C}|$ being number of object classes.

We further extend the above image-level data with objectinstance annotations by following the few-shot object detection formulation $[24,62,66]$. We split the classes into two disjoint sets: base classes $\mathcal{C}_{\text {base }}$ and novel classes $\mathcal{C}_{\text {novel }}$; $\mathcal{C}_{\text {base }} \cap \mathcal{C}_{\text {novel }}=\emptyset$. For base classes, we have abundant instance data $\mathcal{D}_{\text {base }}=\left\{\left(\mathbf{x}_{i}, \mathbf{c}_{i}, \mathbf{y}_{i}\right)\right\}$, where $\mathbf{x}_{i}$ is an input image, $\mathbf{c}_{i}=\left\{c_{i, j}\right\}$ are class labels, $\mathbf{y}_{i}=\left\{\mathbf{b b o x}_{i, j}\right\}$ or $\mathbf{y}_{i}=\left\{\mathbf{s}_{i, j}\right\}$ are corresponding bounding boxes and/or masks for each instance $j$ in image $i$. For novel classes, we have limited instance data $\mathcal{D}_{\text {novel }}=\left\{\left(\mathbf{x}_{i}, \mathbf{c}_{i}, \mathbf{y}_{i}\right)\right\}$, where a $k$-shot detection / segmentation data only has $k$ bounding boxes / masks for each novel class in $\mathcal{C}_{\text {novel }}$. Note, for semi-supervised zero-shot, $k=0$ and $\mathcal{D}_{\text {novel }}=\emptyset$.

\section{Approach}

We propose a single unified framework that leverages the weak image-level supervision for object detection / segmentation in any-shot setting. That is, our proposed approach can seamlessly incorporate arbitrary levels of instance-level supervision without the need to alter the architecture.

Our proposed framework builds upon the Faster R-CNN 


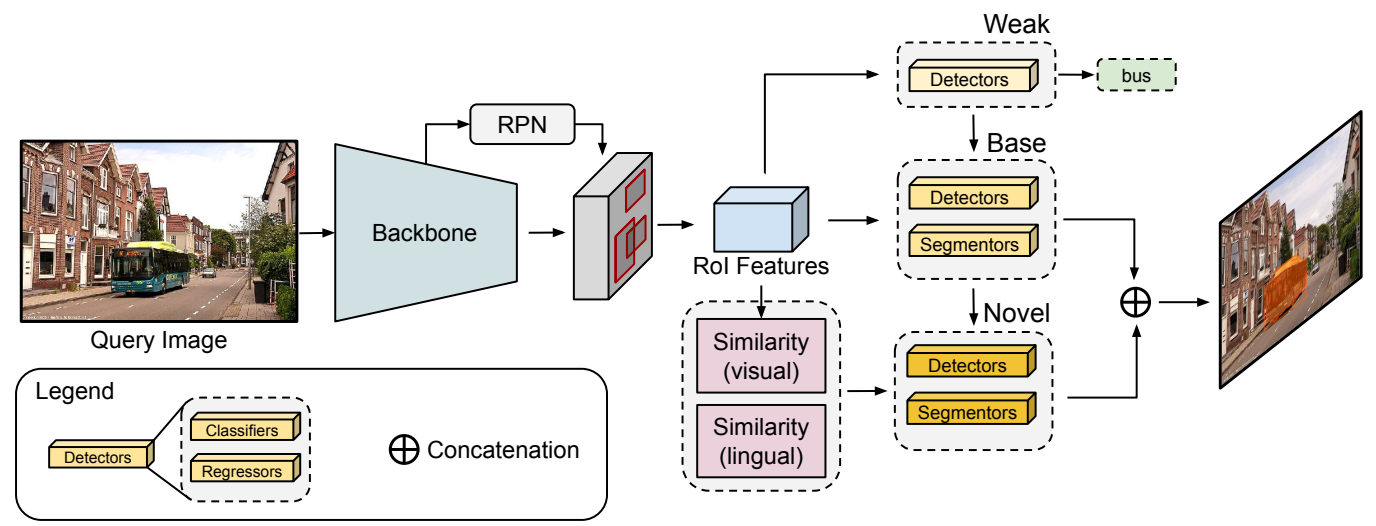

Figure 2: Overall Architecture. We form detectors/segmentors of base classes as a refinement on top of the weak detectors. The detectors/segmentors of novel classes utilize a similarity weighed transfer from the base class refinements. In $k$-shot setting, (few) novel class instance annotations are incorporated through direct adaptation of the resulting novel detectors/segmenters through fine-tuning. The similarity is a combination of lingual and visual similarity (pink boxes). All detectors are built on top of Faster/Mask RCNN architecture which comprises of classification and regression heads with shared backbone (in cyan) and simultaneously trained region proposal network (RPN).

[42] / Mask R-CNN [20] architecture. Faster R-CNN [42] utilizes a two-stage pipeline in order to perform object detection. The first stage uses a region proposal network (RPN) to generate class-agnostic object region proposals $\left\{\operatorname{rbox}_{i, j}\right\}$ for image $i$. The second stage is a detection network (Fast R-CNN [19]) that performs RoI pooling, forming feature vector $\mathbf{z}_{i, j}=\operatorname{RoIAlign}\left(\mathbf{x}_{i}, \operatorname{rbox}_{i, j}\right)$ for proposal $j$ in image $i$, and learns to classify this RoI feature vector $\mathbf{z}$ (we drop proposal and image indexing for brevity for remainder of the section) into one of the object classes and refine the bounding box proposals using a class-aware regressors. Conceptually, an R-CNN object detector can be thought of as a combination of a classifier and regressor (see Figure 2). Mask R-CNN [20] is a simple extension to the Faster $\mathrm{R}-\mathrm{CNN}$ framework, wherein an additional head is utilized in the second stage to predict the instance segmentation masks.

Figure 2 details the proposed architecture. The model consists of two branches: i) the weakly-supervised branch that trains detectors $\hat{c}=\operatorname{softmax}\left(f_{\mathrm{W}_{\text {weak }}}(\mathbf{z})\right)$ using imagelevel supervision $\mathcal{D}^{\text {class }}$, and ii) a supervised branch that uses detection data $\mathcal{D}_{\text {base }} / \mathcal{D}_{\text {novel }}$ to learn a refinement mapping from the weak detector to category-aware classifiers, regressors, and segmentors $f_{\mathbf{W}^{*}}(\mathbf{z}) ; * \in\{$ cls, reg, seg $\}$, which are used in the second stage of Faster / Mask R-CNN. Note that weak detectors simply output the proposal box of the pooled feature vector as the final location $\hat{\mathbf{y}}=\operatorname{rbox}$; while refined detectors are able to regress a better box. Here $f_{\mathbf{W}}(\cdot)$ is a learned neural network function parametrized by W. We jointly train both branches and the RPN, and learning is divided into two stages: base-training and fine-tuning ${ }^{2}$.

Base-training: During base-training, instances from $\mathcal{D}_{\text {base }}$ are used to obtain a detector / segmentation network for the base classes $\mathcal{C}_{\text {base }}$. Specifically, for each $b \in \mathcal{C}_{\text {base }}$,

\footnotetext{
${ }^{2}$ We use the nomenclature introduced in [24].
}

category-aware classifiers and regressors for the base classes are formulated as additive refinements to their corresponding weak counterparts. For region classifiers this takes the form $\begin{aligned} \text { of: } \hat{c}=\underset{\mathcal{C}_{\text {base }}}{\arg \max }[\operatorname{softmax} & \left.\left(f_{\mathbf{W}_{\text {base }}^{\text {cls }}}(\mathbf{z})\right)\right], \text { where } \\ & f_{\mathbf{W}_{\text {base }}^{\text {cls }}}(\mathbf{z})=f_{\mathbf{W}_{\text {base }}^{\text {weak }}}(\mathbf{z})+f_{\Delta \mathbf{W}_{\text {base }}^{\text {cls }}}(\mathbf{z}),\end{aligned}$

where $f_{\Delta \mathbf{W}_{\text {base }}^{\text {cls }}}(\mathbf{z})$ is a zero-initialized residual to the logits of the weakly supervised detector. The regressed object location is similarly defined as:

$$
\hat{\mathbf{y}}=\operatorname{rbox}+f_{\mathbf{W}_{\text {base }}^{\text {reg }}}(\mathbf{z}) .
$$

Finally, as there is no estimate for the segmentation masks in the first stage of Mask R-CNN [20], $\hat{\mathbf{y}}=f_{\mathbf{W}_{\text {base }}^{\text {seg }}}(\mathbf{z})$ is learned directly from the base annotations.

Novel fine-tuning $(k>0)$ : In the fine-tuning phase, the detectors / segmentors of the base classes are used to transfer information to the classes in $\mathcal{C}_{\text {novel }}$. The network is also fine-tuned on $\mathcal{D}_{\text {novel }}$, which, for a value of $k$, contains $k$ bounding boxes / masks for novel and base classes. Here we consider the case of $k>0$; we later address $k=0$ case, which does not require fine-tuning. The key insight of our approach is to use additional visual and lingual similarities between the novel and base classes to enable effective transfer of the network onto the novel classes under varying degrees of supervision. Contrary to existing work $[22,55,26]$ that only consider information from base category-aware classifiers, our approach additionally learns a mapping from base category-aware regressors and segmentors to obtain more accurate novel counterparts. For a specific proposal rbox with features $\mathbf{z}$, let $\mathbf{S}(\mathbf{z}) \in \mathbb{R}^{\left|\mathcal{C}_{\text {novel }}\right| \times\left|\mathcal{C}_{\text {base }}\right|}$ denote similarity between base classes and novel classes. The dependence on $\mathbf{z}$ stems from visual component of the similarity and is discussed in Section 4.2. Given this, for each proposal $\mathbf{z}$, the 
category-aware classifier for the novel classes is obtained as follows: $\hat{c}=\underset{\mathcal{C}_{\text {novel }}}{\arg \max }\left[\operatorname{softmax}\left(f_{\mathbf{W}_{\text {novel }}^{\text {cls }}}(\mathbf{z})\right)\right]$, where $f_{\mathbf{W}_{\text {novel }}^{\text {cls }}}(\mathbf{z})$ can be written as,

$$
\underbrace{f_{\mathbf{W}_{\text {nowel }}^{\text {weak }}}(\mathbf{z})}_{\text {weak-detectors }}+\underbrace{\mathbf{S}(\mathbf{z})^{T} f_{\Delta \mathbf{W}_{\text {base }}^{\text {cls }}}(\mathbf{z})}_{\begin{array}{c}
\text { instance-level transfer } \\
\text { from base classes }
\end{array}}+\underbrace{f_{\Delta \mathbf{W}_{\text {novel }}^{\text {cls }}}(\mathbf{z})}_{\begin{array}{c}
\text { instance-level } \\
\text { direct adaptation }
\end{array}}
$$

where $\mathbf{S}(\mathbf{z})=$ softmax $\left(\mathbf{S}^{\text {lin }} \odot \mathbf{S}^{\text {vis }}(\mathbf{z})\right)$, and $\odot$ denotes broadcast of vector similarity $\mathbf{S}^{\text {vis }}(\mathbf{z}) \in \mathbb{R}^{\left|\mathcal{C}_{\text {base }}\right|}$ followed by element-wise product with lingual similarity $\mathbf{S}^{\text {lin }} \in \mathbb{R}^{\left|\mathcal{C}_{\text {novel }}\right| \times\left|\mathcal{C}_{\text {base }}\right|}$. The interpretation of Eq.(3) is actually rather simple - we first refine the weak detectors for novel classes by similarity weighted additive refinements from base classes (e.g., novel class motorbike may relay on base class bicycle for refinement; illustrations in Section $\mathrm{H}$ of the appendix.), denoted by "instance-level transfer from base classes"; we then further directly adapt the resulting detector (last term) with few instances of the novel class. Similarly, for each $\mathbf{z}$, the novel class object regressor can be obtained as,

$$
\begin{aligned}
\hat{\mathbf{y}} & =\mathbf{r b o x}+f_{\mathbf{W}_{\text {novel }}^{\text {reg }}}(\mathbf{z}) \\
& =\mathbf{r b o x}+\underbrace{\mathbf{S}^{T}(\mathbf{z}) f_{\mathbf{W}_{\text {base }}^{\text {reg }}}(\mathbf{z})}_{\begin{array}{c}
\text { instance-level transfer } \\
\text { from base classes }
\end{array}}+\underbrace{f_{\Delta \mathbf{W}_{\text {novel }}^{\text {reg }}}(\mathbf{z})}_{\begin{array}{c}
\text { instance-level } \\
\text { direct adaptation }
\end{array}}
\end{aligned}
$$

Finally, the segmentation head $f_{\mathbf{W}_{\text {novel }}^{\text {seg }}}(\mathbf{z})$ can be obtained as follows (additional details in appendix Section A),

$$
\hat{\mathbf{y}}=f_{\mathbf{W}_{\text {novel }}^{\text {seg }}}(\mathbf{z})=\underbrace{\mathbf{S}^{T}(\mathbf{z}) f_{\mathbf{W}_{\text {base }}^{\text {seg }}}(\mathbf{z})}_{\begin{array}{c}
\text { instance-level transfer } \\
\text { from base classes }
\end{array}}+\underbrace{f_{\Delta \mathbf{W}_{\text {novel }}^{\text {seg }}}(\mathbf{z})}_{\begin{array}{c}
\text { instance-level } \\
\text { direct adaptation }
\end{array}}
$$

Semi-supervised zero-shot $(k=0)$ : As we mentioned previously, our model is also readily applicable when $\mathcal{C}_{\text {novel }}=$ $\emptyset$. This is a special case of the formulation above, where fine-tuning is not necessary or possible, and we only rely on base training and apply novel class evaluation procedure. The predictions for novel classes can be done as in Eq.(3), Eq.(4), and Eq.(5), but omitting the "instance-level direct adaptation" term in all three cases.

\subsection{Weakly-Supervised Detector}

As mentioned earlier, our approach leverages detectors trained on image level annotations to learn a mapping to supervised detectors/segmentors. We highlight that our approach is agnostic to the method used to train the weaklysupervised detector, and most of the existing approaches $[2,5,53,54]$ can be integrated into our framework. We, however, use the Online Instance Classifer Refinemnet (OICR) architecture proposed by Tang et al. [54] due to its simple architecture. OICR has $R$ "refinement" modules $f_{\mathbf{W}_{r}^{\text {weak }}}(\mathbf{z})$ that progressively improve the detection quality. These individual "refinement" modules are combined to obtain the final prediction as follows,

$\hat{\mathbf{a}}=\operatorname{softmax}\left[f_{\mathbf{W}_{\text {weak }}}(\mathbf{z})\right]=\operatorname{softmax}\left[\frac{1}{R} \sum_{r} f_{\mathbf{W}_{r}^{\text {weak }}}(\mathbf{z})\right]$

We use the same loss formulation $\mathcal{L}^{\text {weak }}(\mathbf{a}, \hat{\mathbf{a}})$ described in [54], which compares predicted (â) and ground truth (a) class labels, to train the OICR module (see Sect. 4.3). For additional details, we refer the reader to [54].

\subsection{Similarity Matrices}

As described in Eq.(3), (4), (5), the key contribution of our approach is the ability to semantically decompose the classifiers, detectors and segmentors of novel classes into their base classes' counterparts. To this end, we define a proposal-aware similarity $\mathbf{S}(\mathbf{z}) \in \mathbb{R}^{\left|\mathcal{C}_{\text {novel }}\right| \times\left|\mathcal{C}_{\text {base }}\right|}$, where each element captures the semantic similarity of novel class $n$ to base class $b$. We assume the similarity matrix $\mathbf{S}(\mathbf{z})$ can be decomposed into two components: lingual $\mathbf{S}^{\text {lin }}$ and visual $\mathbf{S}^{\text {vis }}(\mathbf{z})$ similarity.

Lingual Similarity: This term captures linguistic similarity between novel and base class labels. The intuition lies in the observation that semantically similar classes often have correlated occurrences in textual data. Therefore, for a novel class $n$ and a base class $b, \mathbf{S}_{n, b}^{l i n}=\mathbf{g}_{n}^{\top} \mathbf{g}_{b}$, where $\mathbf{g}_{n}$ and $\mathbf{g}_{b}$ are 300-dimensional GloVe [37] vector embeddings for $n$ and $b$ respectively ${ }^{3}$.

Visual Similarity: Complementary to the lingual component, this proposal-aware similarity models the visual likeness of a proposal $\mathbf{z}$ to objects from the base classes. To this end, for each $\mathbf{z}$, we use the normalized predictions â of the weak detector $f_{\mathbf{W}_{\text {weak }}}(\mathbf{z})$, as described in Eq. (6), as a proxy for the likelihood of $\mathbf{z}$ belonging to a base class $b$. Specifically, let $\hat{\mathbf{a}}_{b}$ be the score corresponding to the base class $b$. For a novel class $n$ and a base class $b$, the visual similarity $\mathbf{S}_{n, b}^{v i s}(\mathbf{z})$ is then defined as,

$$
\mathbf{S}_{n, b}^{v i s}(\mathbf{z})=\frac{\hat{\mathbf{a}}_{b}}{\sum_{b} \hat{\mathbf{a}}_{b}}
$$

Note that computing this visual similarity does not require learning additional parameters. Rather, it is just a convenient by-product of training our model. As a result, this similarity can be efficiently computed. Our proposed formulation for visual similarity, in its essence, is similar to the one used in [55]. However, [55] use image-level scores aggregated over validation set, lacking ability to adapt to a specific proposal.

We would also like to highlight that our framework is extremely flexible and can easily utilize any additional information, similar to [26], to obtain a more accurate semantic

\footnotetext{
${ }^{3}$ For class names that contain multiple words, we average individual GloVe word embeddings.
} 
decomposition $\mathbf{S}(\mathbf{z})$. However, as computing these might require the use of additional datasets and pre-trained models, we refrain from incorporating them into our model.

\subsection{Training}

We now describe the optimization objective used to train our proposed approach in an end-to-end fashion. During base training, the objective can be written as,

$$
\mathcal{L}^{t}=\mathcal{L}^{r c n n}+\alpha \mathcal{L}^{\text {weak }}
$$

where $\mathcal{L}^{r c n n}$ is the Faster/Mask R-CNN [20, 42] objective, and $\mathcal{L}^{\text {weak }}$ is the OICR [54] objective; $\alpha=1$ is the weighting hyperparameter. In fine-tuning, we refine the model only using $\mathcal{L}^{r c n n}$. Fine-tuning only effects last term of Eq.(3), (4), and (5), while everything else is optimized using base training objective. Further implementation details are in Section $\mathrm{B}$ of the appendix. We will make all code and pre-trained models publicly available.

\section{Experiments}

We evaluate our approach against related methods in the semi-supervised and few-shot domain. Comparison against work in the weakly-supervised object detection literature is provided in appendix Section E.

\subsection{Semi-supervised Object Detection}

Datasets. We evaluate the performance of our framework on MSCOCO [32] 2015 and 2017 datasets. Similar to [17, 26], we divide the 80 object categories into 20 base and 60 novel classes, where the base classes are identical to the 20 VOC [14] categories. For our model and the baselines, we assume image-level supervision for all 80 classes, whereas instancelevel supervision is only available for 20 base classes. For few-shot experiments $(k>0)$ we additionally assume $k$ instance-level annotations for the novel classes.

Semi-supervised zero-shot $(k=0)$. Table 1 compares the performance of our proposed approach against the most relevant semi-supervised zero-shot $(k=0)$ methods [22, 23, 26, 55] on novel classes. As an upper-bound, we also show the performance of a fully-supervised model. To ensure fair comparison, we follow the experimental setting in the strongest baseline DOCK [26], and borrow the performance for $[22,23,55]$ from their paper. All models are trained using the same backbone: VGG-CNN-F [6] which is pretrained on the ImageNet classification dataset [11]. Also, similar to [26], we use the MCG [38] proposals instead of training the RPN. The models are evaluated using $\mathrm{mAP}$ at IoU threshold 0.5 denoted as $\mathrm{AP}_{50}$.

UniT beats the closest baseline, DOCK [26], by a significant margin $\left(\sim 16 \%\right.$ on $\left.\mathrm{AP}_{50}\right)$, despite DOCK using more sophisticated similarity measures for knowledge transfer, which require additional data from VOC [14], Visual Genome [25], and SUN [65] datasets. As DOCK only transfers knowledge from base class classifiers, this difference

\begin{tabular}{lcccc}
\hline \multicolumn{1}{c}{ Method } & $\mathrm{AP}_{50}$ & $\mathrm{AP}_{S}$ & $\mathrm{AP}_{M}$ & $\mathrm{AP}_{L}$ \\
\hline LSDA [22] & 4.6 & 1.2 & 5.1 & 7.8 \\
LSDA+Semantic [55] & 4.7 & 1.1 & 5.1 & 8.0 \\
LSDA+MIL [23] & 5.9 & 1.5 & 8.3 & 10.7 \\
DOCK [26] & 14.4 & 2.0 & 12.8 & 24.9 \\
\hline UniT (Ours) & $\mathbf{1 6 . 7}$ & $\mathbf{3 . 2}$ & $\mathbf{1 6 . 6}$ & $\mathbf{2 7 . 3}$ \\
\hline Full Supervision [26] & 25.2 & 5.8 & 26.0 & 41.6 \\
\hline
\end{tabular}

Table 1: Comparison to semi-supervised zero-shot. All models are trained on VGG-CNN-F [6] backbone.

\begin{tabular}{lccccc}
\hline Method / Shots $(k)$ & 12 & 33 & 55 & 76 & 96 \\
\hline NOTE-RCNN [17] & 14.1 & 14.2 & 17.1 & 19.8 & 19.9 \\
UniT (Ours) & $\mathbf{1 4 . 7}$ & $\mathbf{1 7 . 4}$ & $\mathbf{1 9 . 3}$ & $\mathbf{2 0 . 9}$ & $\mathbf{2 2 . 1}$ \\
\hline
\end{tabular}

Table 2: Comparison to semi-supervised few-shot. All models are trained on Inception-ResNet-v2 [52] backbone. Mean Average Precision (mAP) on novel classes averaged over IoU thresholds in $[0.5: 0.05: 0.95]$ is reported.

in performance can be attributed to UniT additionally effectively transferring knowledge from base class regressors onto novel class regressors (Eq. 4). It can also be noted that our work can be considered complimentary to DOCK, as we can easily integrate their richer similarity measures into our framework by modifying $\mathbf{S}(\mathbf{z})$ (Sec. 4.2).

Semi-supervised few-shot $(k>0)$. Table 2 compares the performance of our method with NOTE-RCNN [17], which is the only relevant baseline under this setting, on novel classes. We follow the experimental setting described in [17], and our model is trained using the same backbone as NOTE-RCNN: Inception-Resnet-V2 [52] pretrained on the ImageNet classification dataset [11], where the RPN is learned from the instance-level base data. Similar to [17], we assume $k$ instance-level annotations for the novel classes, where $k \in\{12,33,55,76,96\}$. To ensure fair comparison, the performance of NOTE-RCNN [17] is taken from their published work ${ }^{4}$. We report mAP on novel classes averaged over IoU thresholds in $[0.5: 0.05: 0.95]$.

UniT outperforms NOTE-RCNN [17] on all values of $k$, providing an improvement of up to $\sim 23 \%$. Contrary to NOTE-RCNN that only trains novel regressors on the $k$ shots, UniT benefits from effectively mapping information from base regressors to novel regressors. In addition, UniT also has the advantage of allowing end-to-end training while simultaneously being simple and interpretable. NOTE-RCNN, on the other hand, employs a complex multi-step bounding box mining framework that takes longer to train on novel classes. Note that, in principle, one could incorporate the box mining mechanism into our framework as well.

${ }^{4}[17]$ visualize their numbers as a plot instead of listing the raw values. As the authors were unreachable, Table 2 lists our best interpretation of the numbers shown in the plot. 


\begin{tabular}{|c|c|c|c|c|c|c|c|c|c|c|c|c|c|c|c|c|c|c|c|}
\hline \multirow[b]{2}{*}{ Method / Shots } & & \multicolumn{6}{|c|}{ Novel Set 1} & \multicolumn{6}{|c|}{ Novel Set 2} & \multicolumn{6}{|c|}{ Novel Set 3} \\
\hline & & 0 & 1 & 2 & 3 & 5 & 10 & 0 & 1 & 2 & 3 & 5 & 10 & 0 & 1 & 2 & 3 & 5 & 10 \\
\hline Joint & FRCN [66] & - & 2.7 & 3.1 & 4.3 & 11.8 & 29.0 & - & 1.9 & 2.6 & 8.1 & 9.9 & 12.6 & - & 5.2 & 7.5 & 6.4 & 6.4 & 6.4 \\
\hline Transfer & FRCN [61] & - & 15.2 & 20.3 & 29.0 & 40.1 & 45.5 & - & 13.4 & 20.6 & 28.6 & 32.4 & 38.8 & - & 19.6 & 20.8 & 28.7 & 42.2 & 42.1 \\
\hline \multirow{4}{*}{ Few-Shot } & Kang et al. [24] & - & 14.8 & 15.5 & 26.7 & 33.9 & 47.2 & - & 15.7 & 15.3 & 22.7 & 30.1 & 39.2 & - & 19.2 & 21.7 & 25.7 & 40.6 & 41.3 \\
\hline & Wang et al. [62] & - & 18.9 & 20.6 & 30.2 & 36.8 & 49.6 & - & 21.8 & 23.1 & 27.8 & 31.7 & 43.0 & - & 20.6 & 23.9 & 29.4 & 43.9 & 44.1 \\
\hline & Yan et al. [66] & - & 19.9 & 25.5 & 35.0 & 45.7 & 51.5 & - & 10.4 & 19.4 & 29.6 & 34.8 & 45.4 & - & 14.3 & 18.2 & 27.5 & 41.2 & 48.1 \\
\hline & Wang et al. [61] & - & 39.8 & 36.1 & 44.7 & 55.7 & 56.0 & - & 23.5 & 26.9 & 34.1 & 35.1 & 39.1 & - & 30.8 & 34.8 & 42.8 & 49.5 & 49.8 \\
\hline Semi+Any Shot & UniT (Ours) & 75.6 & 75.7 & 75.8 & 75.9 & 76.1 & 76.7 & 56.9 & 57.2 & 57.4 & $\mathbf{5 7 . 9}$ & 58.2 & 63.0 & 67.5 & 67.6 & 68.1 & 68.2 & 68.6 & 70.0 \\
\hline Fully-supervised & FRCN & & & & 84.71 & & & & & & 82.89 & & & & & & .57 & & \\
\hline
\end{tabular}

Table 3: Few-shot object detection on VOC. FRCN = Faster R-CNN with ResNet-101 backbone. Mean AP Fo $_{\text {reported on }}$ novel classes; performance on base classes is reported in supplementary Section I.

\begin{tabular}{cccccccc}
\hline \#Shots & & $\mathrm{AP}$ & $\mathrm{AP}_{50}$ & $\mathrm{AP}_{75}$ & $\mathrm{AP}_{S}$ & $\mathrm{AP}_{M}$ & $\mathrm{AP}_{L}$ \\
\hline$k=0$ & UniT (Ours) & 18.9 & 36.1 & 17.5 & 8.7 & 20.4 & 27.6 \\
\hline \multirow{5}{*}{$k=10$} & Transfer: FRCN [66] & 6.5 & 13.4 & 5.9 & 1.8 & 5.3 & 11.3 \\
& Kang et al. [24] & 5.6 & 12.3 & 4.6 & 0.9 & 3.5 & 10.5 \\
& Wang et al. [62] & 7.1 & 14.6 & 6.1 & 1.0 & 4.1 & 12.2 \\
& Yan et al. [66] & 8.7 & 19.1 & 6.6 & 2.3 & 7.7 & 14.0 \\
& Wang et al. [61] & 10.0 & - & 9.3 & - & - & - \\
& UniT (Ours) & $\mathbf{2 1 . 7}$ & $\mathbf{4 0 . 8}$ & $\mathbf{2 0 . 6}$ & $\mathbf{9 . 1}$ & $\mathbf{2 3 . 8}$ & $\mathbf{3 1 . 3}$
\end{tabular}

Table 4: Few-shot object detection on COCO. FRCN=Faster R-CNN with ResNet-50 backbone. Complete table is in supplementary Section C.

\subsection{Few-shot Object Detection and Segmentation}

Datasets. We evaluate our models on VOC 2007 [14], VOC 2012 [13], and MSCOCO [32] as used in the previous fewshot object detection and segmentation works [24, 61, 62, 66]. For both detection and segmentation, we consistently follow the data splits introduced and used in [24, 66]. In case of VOC, we use VOC 07 test set (5k images) for evaluation and VOC $07+12$ trainval sets (16.5k images) for training. The 20 object classes are divided into 15 base and 5 novel classes with 3 different sets of class splits. For novel classes, we use images made available by Kang et al. [24] for $k$-shot fine-tuning. We report mean Average Precision (mAP) on novel classes and use a standard IoU threshold of 0.5 [14]. Similarly, for the MSCOCO [32] dataset, consistent with [24] we use $5 \mathrm{k}$ images from the validation set for evaluation and the remaining $115 \mathrm{k}$ trainval images for training. We assign 20 object classes from VOC as the novel classes and remaining 60 as the base classes. We report the standard evaluation metric on $\mathrm{COCO}$ [42].

PASCAL VOC Detection. Table 3 summarizes the results on VOC for three different novel class splits with different $k$-shot settings. Following [61, 66], we assume Faster R-CNN [42] with ResNet-101 [21] as the backbone which is pre-trained on ImageNet-1k [44]. UniT outperforms the related state-of-the-art methods on all values of $k$, including the scenario where no instance-level supervision for novel classes is available $(k=0)$, showing the effectiveness of transfer from base to novel classes. As UniT uses additional weak image-level data for novel classes, this is not an equiv-

\begin{tabular}{|c|c|c|c|c|c|c|c|c|}
\hline \#Shots & Method & & AP & $\mathrm{AP}_{50}$ & $\mathrm{AP}_{75}$ & $\mathrm{AP}_{S}$ & $\mathrm{AP}_{M}$ & $\mathrm{AP}_{L}$ \\
\hline \multirow{2}{*}{$k=0$} & \multirow{2}{*}{ UniT (Ours) } & Box & 20.2 & 36.8 & 19.5 & 8.5 & 20.9 & 28.9 \\
\hline & & Mask & 17.6 & 32.7 & 17.0 & 5.6 & 17.6 & 27.7 \\
\hline \multirow{4}{*}{$k=10$} & \multirow{2}{*}{ Yan et al. [66] } & Box & 5.6 & 14.2 & 3.0 & 2.0 & 6.6 & 8.8 \\
\hline & & Mask & 4.4 & 10.6 & 3.3 & 0.5 & 3.6 & 7.2 \\
\hline & \multirow{2}{*}{ UniT (Ours) } & Box & $\overline{22.8}$ & 41.6 & 21.9 & 9.4 & 24.4 & 32.3 \\
\hline & & Mask & 20.5 & 38.6 & 19.7 & 6.0 & 20.5 & 31.8 \\
\hline
\end{tabular}

Table 5: Few-shot instance segmentation on COCO. Complete table is in supplementary Section D.

alent comparison (see Sec. 5.3 comparisons under similar annotation budget). But we want to highlight that such data is readily available, much cheaper to obtain [4], and leads to a significant improvement in performance.

MS-COCO Detection. Table 4 describes the results on COCO dataset. Similar to [66, 61], we use ImageNet [11] pretrained ResNet-50 [21] as the backbone. We observe similar trends as above. In addition, we note that our performance consistently increases with the value of $k$ even on larger datasets, showing that UniT is effective and flexible in scaling with the degree of instance-level supervision ranging from zero to a few. The full table is in appendix Section C. Figure 3 shows qualitative results, indicating our method is able to correctly detect novel classes.

MS-COCO Segmentation. Table 5 summarizes the results. Similar to [66], we choose an ImageNet[11] pretrained ResNet-50 [21] backbone. UniT consistently improves over [66], demonstrating that our approach is not limited to bounding boxes, and is able to generalize over the type of downstream structured label by effectively transferring information from base segmentations to novel segmentations. The full table is provided in appendix Section D. Figure 3 shows some qualitative results on $k=0$ for novel classes.

Ablation. A complete ablation study on MSCOCO [32] is provided in appendix Section G. We report performance on the novel split used by [66], starting with only weak detectors and progressively adding the terms in Eq.(1), (3), (4), and (5). Weighting with visual and lingual similarity results in $+1.4 \mathrm{AP}_{50}$ improvement (Eq. (3)), transfer from 


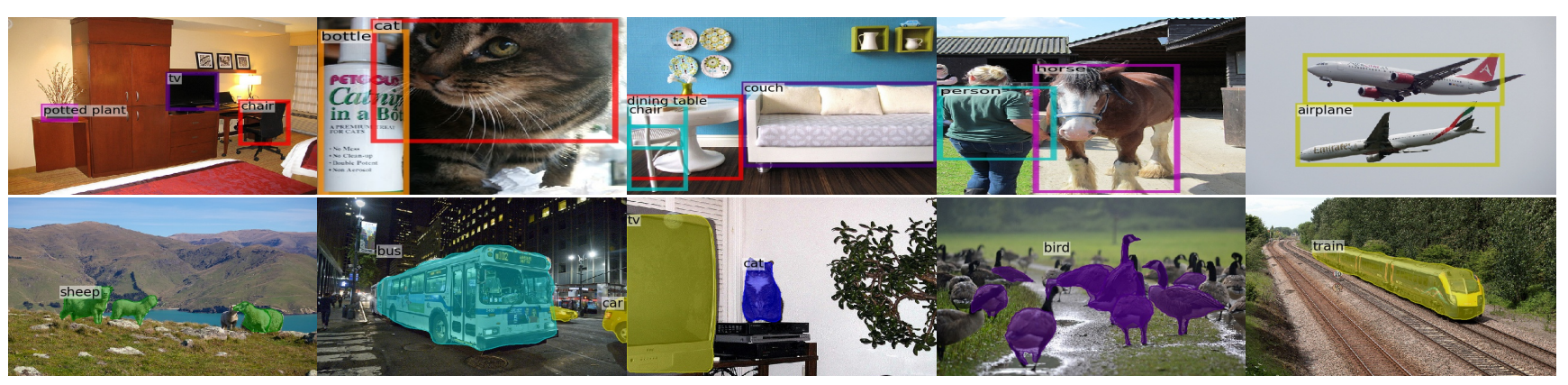

Figure 3: Qualitative Visualizations. Semi-supervised zero-shot $(k=0)$ detection (top) and instance segmentation (bottom) performance on novel classes in MS-COCO (color = object category). Additional visualizations in Section J of the appendix.

base regressors (Eq. (4)) provides an additional $+7 \mathrm{AP}_{50}$ imrovement. Finally, transfer from base class segmentations (Eq. (5)) leads to an added gain of +7.5 on mask $\mathrm{AP}_{50}$.

\subsection{Limited Annotation Budget}

Compared to approaches in the few-shot detection (and segmentation) domain like [24, 61, 62, 66], UniT assumes additional image-level annotations for novel classes. We argue this is a reasonable assumption considering that such annotations are readily available in abundance for thousands of object classes $(\sim 22 \mathrm{~K}$ in ImageNet [11] and $\sim 20 \mathrm{~K}$ in Open Image v4 dataset [28]). Experiments in Section 5.2 further highlight the performance improvements possible by using such inexpensive data. However, this raises an interesting question as to what form of supervision could be more valuable, if one is to collect it. To experiment with this, we conceptually impose an annotation budget that limits the number of novel class image-level annotations our approach can use. For object detection on VOC [13], we assume 7 image-level annotations can be generated in the same time as 1 instance-level annotation. The 7 conversion factor between object instance labels and weakly-supervised image-level labels is motivated by the timings reported in [4] and is a conservative estimate (additional details in appendix Section F) ${ }^{5}$. Therefore, for each value of $k$ in a few-shot setup, we train a variant of UniT that assumes only $7 \times k$ image-level annotations for novel classes, which is referred to as $\mathrm{UniT}_{\text {budget }=k}$. We then compare the zero-shot performance of $\mathrm{UniT}_{\text {budget }=k}$ against the corresponding $k$-shot generalized object detection benchmarks ${ }^{6}$ reported in [61]. This setting allows for an apples-to-apples comparison with the baselines, while simultaneously enabling the analysis of the relative importance of image-level annotations when compared to instance-level annotations.

Please refer to Section 5.2 for details on the dataset and setup. Table 6 summarizes the results on VOC for three different novel class splits with different $k$-shot settings. For

\footnotetext{
${ }^{5}$ This factor is expected to be higher in practice, as we don't consider situations where boxes/masks are rejected and need to be redrawn [35].

${ }^{6}$ These benchmarks use multiple random splits as opposed to curated splits used in [24] and Table 3. As per [61], this helps reduce variance.
}

\begin{tabular}{ccccc}
\hline \#Shots & Method & Split 1 & Split 2 & Split 3 \\
\hline \multirow{4}{*}{1} & Kang et al. [24] & $14.2 \pm 1.7$ & $12.3 \pm 1.9$ & $12.5 \pm 1.6$ \\
& Wang et al. [61] & $25.3 \pm 2.2$ & $\mathbf{1 8 . 3} \pm \mathbf{2 . 4}$ & $17.9 \pm 2.0$ \\
& UniT $_{\text {budget }=1 \text { (Ours) }}$ & $\mathbf{2 8 . 3} \pm \mathbf{2 . 0}$ & $17.0 \pm 1.9$ & $\mathbf{2 6 . 2} \pm \mathbf{2 . 5}$ \\
\hline \multirow{3}{*}{5} & Kang et al. [24] & $36.5 \pm 1.4$ & $31.4 \pm 1.5$ & $33.8 \pm 1.4$ \\
& Wang et al. [61] & $47.9 \pm 1.2$ & $34.1 \pm 1.4$ & $40.8 \pm 1.4$ \\
& UniT $_{\text {budget }=5}$ (Ours) & $\mathbf{5 0 . 9} \pm \mathbf{1 . 4}$ & $\mathbf{3 6 . 2} \pm \mathbf{1 . 7}$ & $\mathbf{4 7 . 4} \pm \mathbf{1 . 2}$ \\
\hline \multirow{2}{*}{10} & Wang et al. [61] & $52.8 \pm 1.0$ & $39.5 \pm 1.1$ & $45.6 \pm 1.1$ \\
& UniT $_{\text {budget }=10}$ (Ours) & $\mathbf{5 9 . 0} \pm \mathbf{1 . 5}$ & $\mathbf{4 0 . 8} \pm \mathbf{1 . 3}$ & $\mathbf{5 2 . 9} \pm \mathbf{1 . 1}$ \\
\hline
\end{tabular}

Table 6: Limited annotation budget. Averaged $\mathrm{AP}_{50}$ for 10 random runs with $95 \%$ confidence interval estimate [61].

accurate results, on each split and $k$-shot, we perform 10 repeated runs of UniT $_{\text {budget }=k}$, selecting a random set of $7 \times k$ weakly-labelled novel class images each time. Following [61], we assume ResNet-101 [21] as the backbone. In table 7, for novel split 1, we further analyse the relative importance of image-level to instance-level annotations. For a fixed budget equivalent to 10 instance-level annotations, we experiment with using different proportions of image and instance-level annotations, and report mean $\mathrm{AP}_{50}$ on novel classes computed across 10 repeated runs.

Even under the constraint of equal budget, $\mathrm{UniT}_{\text {budget }}=k$ outperforms the state-of-the-art [61] on multiple splits. This highlights three key observations: i) weak image-level supervision, which is cheaper to obtain [4], provides a greater 'bang-for-the-buck' when compared to instance-level supervision, ii) our structured transfer from base classes is effective even when the amount of novel class supervision is limited, and iii) from Table 7, in a low-shot and fixed budget setting, it is more beneficial to just use weak supervision (instead of some combination of both). Furthermore, as our approach is agnostic to the type of weak detector used, employing better weak detectors like [53,2] could further improve the performance of $\mathrm{UniT}_{\text {budget }}=k$.

\section{Discussion and Conclusion}

We propose an intuitive semi-supervised model that is applicable to a range of supervision: from zero to a few instance-level samples per novel class. For base classes, our model learns a mapping from weakly-supervised to fullysupervised detectors/segmentors. By leveraging similarities 


\begin{tabular}{cccc}
\hline Method & $\begin{array}{c}\text { Weak } \\
\text { Anno.(\%) }\end{array}$ & $\begin{array}{c}\text { Instance } \\
\text { Anno.(\%) }\end{array}$ & $\mathrm{AP}_{50}$ \\
\hline Wang et al. $[61]+10$-Shots & 0 & 100 & $52.8 \pm 1.0$ \\
UniT $_{\text {budget }=1}+9$-Shots & 10 & 90 & $49.2 \pm 0.6$ \\
UniT $_{\text {budget }=5}+5$-Shots & 50 & 50 & $54.0 \pm 0.8$ \\
UniT $_{\text {budget }=10}+$ 0-Shots & 100 & 0 & $\mathbf{5 9 . 0} \pm \mathbf{1 . 5}$ \\
\hline
\end{tabular}

Table 7: Using different annotation proportions. For the same budget, we vary the amount of image/instance level annotation. Averaged $\mathrm{AP}_{50}$ for 10 random runs with $95 \%$ confidence interval estimate of the mean values [61] is shown.

between the novel and base classes, we transfer those mappings to obtain detectors/segmentors for novel classes; refining them with a few novel class instance-level annotated samples, if available. This versatile paradigm works significantly better than traditional semi supervised and few-shot detection and segmentation methods.

\section{Acknowledgments and Disclosure of Funding}

This work was funded, in part, by the Vector Institute for AI, Canada CIFAR AI Chair, NSERC CRC and an NSERC DG and Discovery Accelerator Grants.

This material is based upon work supported by the United States Air Force Research Laboratory (AFRL) under the Defense Advanced Research Projects Agency (DARPA) Learning with Less Labels (LwLL) program (Contract No.FA875019-C-0515). The views and conclusions contained herein are those of the authors and should not be interpreted as necessarily representing the official policies or endorsements, either expressed or implied, of DARPA or the U.S. Government.

Resources used in preparing this research were provided, in part, by the Province of Ontario, the Government of Canada through CIFAR, and companies sponsoring the Vector Institute www. vectorinstitute. ai/ \#partners.

Additional hardware support was provided by John R. Evans Leaders Fund CFI grant and Compute Canada under the Resource Allocation Competition award.

Finally, we would like to sincerely thank Bicheng Xu, Issam Laradji and Prof. Frank Wood for valuable feedback and discussions.

\section{References}

[1] Marcin Andrychowicz, Misha Denil, Sergio Gomez, Matthew W Hoffman, David Pfau, Tom Schaul, Brendan Shillingford, and Nando De Freitas. Learning to learn by gradient descent by gradient descent. In Advances in neural information processing systems, pages 3981-3989, 2016. 2

[2] Aditya Arun, CV Jawahar, and M Pawan Kumar. Dissimilarity coefficient based weakly supervised object detection. In Proceedings of the IEEE Conference on Computer Vision and Pattern Recognition, pages 9432-9441, 2019. 1, 3, 5, 8, 13, 14,15
[3] Ankan Bansal, Karan Sikka, Gaurav Sharma, Rama Chellappa, and Ajay Divakaran. Zero-shot object detection. In Proceedings of the European Conference on Computer Vision (ECCV), pages 384-400, 2018. 1, 2, 3

[4] A. Bearman, O. Russakovsky, V. Ferrari, and L. Fei-Fei. Whats the point: Semantic segmentation with point supervision. In $E C C V, 2016.1,7,8,14,15$

[5] Hakan Bilen and Andrea Vedaldi. Weakly supervised deep detection networks. In Proceedings of the IEEE Conference on Computer Vision and Pattern Recognition, pages 28462854, 2016. 1, 3, 5, 13, 14, 15

[6] Ken Chatfield, Karen Simonyan, Andrea Vedaldi, and Andrew Zisserman. Return of the devil in the details: Delving deep into convolutional nets. BMVC, 2014. 6

[7] Hao Chen, Yali Wang, Guoyou Wang, and Yu Qiao. Lstd: A low-shot transfer detector for object detection. In ThirtySecond AAAI Conference on Artificial Intelligence, 2018. 2

[8] Liang-Chieh Chen, Alexander Hermans, George Papandreou, Florian Schroff, Peng Wang, and Hartwig Adam. Masklab: Instance segmentation by refining object detection with semantic and direction features. In Proceedings of the IEEE Conference on Computer Vision and Pattern Recognition, pages 4013-4022, 2018. 1

[9] Ramazan Gokberk Cinbis, Jakob Verbeek, and Cordelia Schmid. Weakly supervised object localization with multifold multiple instance learning. IEEE transactions on pattern analysis and machine intelligence, 39(1):189-203, 2016. 3

[10] Berkan Demirel, Ramazan Gokberk Cinbis, and Nazli IkizlerCinbis. Zero-shot object detection by hybrid region embedding. arXiv preprint arXiv:1805.06157, 2018. 3

[11] J. Deng, W. Dong, R. Socher, L.-J. Li, K. Li, and L. Fei-Fei. ImageNet: A Large-Scale Hierarchical Image Database. In CVPR09, 2009. 6, 7, 8, 15

[12] Ali Diba, Vivek Sharma, Ali Pazandeh, Hamed Pirsiavash, and Luc Van Gool. Weakly supervised cascaded convolutional networks. In Proceedings of the IEEE conference on computer vision and pattern recognition, pages 914-922, 2017. 1, 3

[13] Mark Everingham, SM Ali Eslami, Luc Van Gool, Christopher KI Williams, John Winn, and Andrew Zisserman. The pascal visual object classes challenge: A retrospective. International journal of computer vision, 111(1):98-136, 2015. 2, 7, 8, 14, 15

[14] Mark Everingham, Luc Van Gool, Christopher KI Williams, John Winn, and Andrew Zisserman. The pascal visual object classes (voc) challenge. International journal of computer vision, 88(2):303-338, 2010. 1, 6, 7, 14, 18

[15] Chelsea Finn, Pieter Abbeel, and Sergey Levine. Modelagnostic meta-learning for fast adaptation of deep networks. In Proceedings of the 34th International Conference on Machine Learning-Volume 70, pages 1126-1135. JMLR. org, 2017. 2

[16] Andrea Frome, Greg S Corrado, Jon Shlens, Samy Bengio, Jeff Dean, Marc' Aurelio Ranzato, and Tomas Mikolov. Devise: A deep visual-semantic embedding model. In Advances in neural information processing systems, pages 2121-2129, 2013. 1, 2, 3 
[17] Jiyang Gao, Jiang Wang, Shengyang Dai, Li-Jia Li, and Ram Nevatia. Note-rcnn: Noise tolerant ensemble renn for semisupervised object detection. In Proceedings of the IEEE international conference on computer vision, pages 9508 9517, 2019. 2, 3, 6

[18] Yan Gao, Boxiao Liu, Nan Guo, Xiaochun Ye, Fang Wan, Haihang You, and Dongrui Fan. C-midn: Coupled multiple instance detection network with segmentation guidance for weakly supervised object detection. In Proceedings of the IEEE International Conference on Computer Vision, pages 9834-9843, 2019. 3

[19] Ross Girshick. Fast r-cnn. In Proceedings of the IEEE international conference on computer vision, pages 1440-1448, 2015. 4, 13

[20] Kaiming He, Georgia Gkioxari, Piotr Dollár, and Ross Girshick. Mask r-cnn. In Proceedings of the IEEE international conference on computer vision, pages 2961-2969, 2017. 1, 4, $6,13,14$

[21] Kaiming He, Xiangyu Zhang, Shaoqing Ren, and Jian Sun. Deep residual learning for image recognition. In Proceedings of the IEEE conference on computer vision and pattern recognition, pages 770-778, 2016. 1, 7, 8, 13, 14, 15

[22] Judy Hoffman, Sergio Guadarrama, Eric S Tzeng, Ronghang Hu, Jeff Donahue, Ross Girshick, Trevor Darrell, and Kate Saenko. Lsda: Large scale detection through adaptation. In Advances in Neural Information Processing Systems, pages 3536-3544, 2014. 1, 2, 3, 4, 6

[23] Judy Hoffman, Deepak Pathak, Trevor Darrell, and Kate Saenko. Detector discovery in the wild: Joint multiple instance and representation learning. In Proceedings of the ieee conference on computer vision and pattern recognition, pages 2883-2891, 2015. 6

[24] Bingyi Kang, Zhuang Liu, Xin Wang, Fisher Yu, Jiashi Feng, and Trevor Darrell. Few-shot object detection via feature reweighting. In The IEEE International Conference on Computer Vision (ICCV), October 2019. 2, 3, 4, 7, 8, 14, 15, 16, 17,18

[25] Ranjay Krishna, Yuke Zhu, Oliver Groth, Justin Johnson, Kenji Hata, Joshua Kravitz, Stephanie Chen, Yannis Kalantidis, Li-Jia Li, David A Shamma, et al. Visual genome: Connecting language and vision using crowdsourced dense image annotations. International journal of computer vision, 123(1):32-73, 2017. 6

[26] Krishna Kumar Singh, Santosh Divvala, Ali Farhadi, and Yong Jae Lee. Dock: Detecting objects by transferring common-sense knowledge. In Proceedings of the European Conference on Computer Vision (ECCV), pages 492-508, 2018. 2, 3, 4, 5, 6

[27] Alina Kuznetsova, Sung Ju Hwang, Bodo Rosenhahn, and Leonid Sigal. Expanding object detector's horizon: Incremental learning framework for object detection in videos. In Proceedings of the IEEE Conference on Computer Vision and Pattern Recognition, pages 28-36, 2015. 3

[28] Alina Kuznetsova, Hassan Rom, Neil Alldrin, Jasper Uijlings, Ivan Krasin, Jordi Pont-Tuset, Shahab Kamali, Stefan Popov, Matteo Malloci, Alexander Kolesnikov, et al. The open images dataset v4. International Journal of Computer Vision, pages $1-26,2020.2,3,8$
[29] Issam H Laradji, David Vazquez, and Mark Schmidt. Where are the masks: Instance segmentation with image-level supervision. In $B M V C, 2019.1,2$

[30] Zhihui Li, Lina Yao, Xiaoqin Zhang, Xianzhi Wang, Salil Kanhere, and Huaxiang Zhang. Zero-shot object detection with textual descriptions. In Proceedings of the AAAI Conference on Artificial Intelligence, volume 33, pages 8690-8697, 2019. 3

[31] Tsung-Yi Lin, Piotr Dollár, Ross Girshick, Kaiming He, Bharath Hariharan, and Serge Belongie. Feature pyramid networks for object detection. In Proceedings of the IEEE conference on computer vision and pattern recognition, pages 2117-2125, 2017. 18

[32] Tsung-Yi Lin, Michael Maire, Serge Belongie, James Hays, Pietro Perona, Deva Ramanan, Piotr Dollár, and C Lawrence Zitnick. Microsoft coco: Common objects in context. In European conference on computer vision, pages 740-755. Springer, 2014. 1, 2, 6, 7, 13, 15, 18

[33] Wei Liu, Dragomir Anguelov, Dumitru Erhan, Christian Szegedy, Scott Reed, Cheng-Yang Fu, and Alexander C Berg. Ssd: Single shot multibox detector. In European conference on computer vision, pages 21-37. Springer, 2016. 1

[34] Dim P Papadopoulos, Alasdair DF Clarke, Frank Keller, and Vittorio Ferrari. Training object class detectors from eye tracking data. In European conference on computer vision, pages 361-376. Springer, 2014. 14

[35] Dim P Papadopoulos, Jasper RR Uijlings, Frank Keller, and Vittorio Ferrari. Training object class detectors with click supervision. In Proceedings of the IEEE Conference on Computer Vision and Pattern Recognition, pages 6374-6383, 2017. 8,15

[36] Adam Paszke, Sam Gross, Francisco Massa, Adam Lerer, James Bradbury, Gregory Chanan, Trevor Killeen, Zeming Lin, Natalia Gimelshein, Luca Antiga, et al. Pytorch: An imperative style, high-performance deep learning library. In Advances in Neural Information Processing Systems, pages 8024-8035, 2019. 13

[37] Jeffrey Pennington, Richard Socher, and Christopher D Manning. Glove: Global vectors for word representation. In Proceedings of the 2014 conference on empirical methods in natural language processing (EMNLP), pages 1532-1543, 2014. 5

[38] Jordi Pont-Tuset, Pablo Arbelaez, Jonathan T Barron, Ferran Marques, and Jitendra Malik. Multiscale combinatorial grouping for image segmentation and object proposal generation. IEEE transactions on pattern analysis and machine intelligence, 39(1):128-140, 2016. 6, 13

[39] Shafin Rahman, Salman Khan, and Fatih Porikli. Zero-shot object detection: Learning to simultaneously recognize and localize novel concepts. In Asian Conference on Computer Vision, pages 547-563. Springer, 2018. 1, 2, 3

[40] Sachin Ravi and Hugo Larochelle. Optimization as a model for few-shot learning. In ICLR, 2017. 2

[41] Joseph Redmon and Ali Farhadi. Yolo9000: better, faster, stronger. In Proceedings of the IEEE conference on computer vision and pattern recognition, pages 7263-7271, 2017. 1, 2

[42] Shaoqing Ren, Kaiming He, Ross Girshick, and Jian Sun. Faster r-cnn: Towards real-time object detection with region 
proposal networks. In Advances in neural information processing systems, pages 91-99, 2015. 1, 2, 4, 6, 7, 13

[43] Zhongzheng Ren, Zhiding Yu, Xiaodong Yang, Ming-Yu Liu, Yong Jae Lee, Alexander G Schwing, and Jan Kautz. Instanceaware, context-focused, and memory-efficient weakly supervised object detection. In Proceedings of the IEEE/CVF Conference on Computer Vision and Pattern Recognition, pages 10598-10607, 2020. 3, 13, 14, 15

[44] Olga Russakovsky, Jia Deng, Hao Su, Jonathan Krause, Sanjeev Satheesh, Sean Ma, Zhiheng Huang, Andrej Karpathy, Aditya Khosla, Michael Bernstein, et al. Imagenet large scale visual recognition challenge. International journal of computer vision, 115(3):211-252, 2015. 1, 3, 7

[45] Arka Sadhu, Kan Chen, and Ram Nevatia. Zero-shot grounding of objects from natural language queries. In Proceedings of the IEEE International Conference on Computer Vision, pages 4694-4703, 2019. 3

[46] Jürgen Schmidhuber. Evolutionary principles in selfreferential learning, or on learning how to learn: the metameta-... hook. PhD thesis, Technische Universität München, 1987. 2

[47] Karen Simonyan and Andrew Zisserman. Very deep convolutional networks for large-scale image recognition. arXiv preprint arXiv:1409.1556, 2014. 13, 14, 15

[48] Jake Snell, Kevin Swersky, and Richard Zemel. Prototypical networks for few-shot learning. In Advances in neural information processing systems, pages 4077-4087, 2017. 2

[49] Hyun Oh Song, Yong Jae Lee, Stefanie Jegelka, and Trevor Darrell. Weakly-supervised discovery of visual pattern configurations. In Advances in Neural Information Processing Systems, pages 1637-1645, 2014. 3

[50] Hao Su, Jia Deng, and Li Fei-Fei. Crowdsourcing annotations for visual object detection. In Workshops at the Twenty-Sixth AAAI Conference on Artificial Intelligence, 2012. 15

[51] Chen Sun, Abhinav Shrivastava, Saurabh Singh, and Abhinav Gupta. Revisiting unreasonable effectiveness of data in deep learning era. In Proceedings of the IEEE international conference on computer vision, pages 843-852, 2017. 1, 2

[52] Christian Szegedy, Sergey Ioffe, Vincent Vanhoucke, and Alex Alemi. Inception-v4, inception-resnet and the impact of residual connections on learning. AAAI, 2017. 6

[53] Peng Tang, Xinggang Wang, Song Bai, Wei Shen, Xiang Bai, Wenyu Liu, and Alan Yuille. Pcl: Proposal cluster learning for weakly supervised object detection. IEEE transactions on pattern analysis and machine intelligence, 42(1):176-191, 2018. 3, 5, 8, 14, 15

[54] Peng Tang, Xinggang Wang, Xiang Bai, and Wenyu Liu. Multiple instance detection network with online instance classifier refinement. In Proceedings of the IEEE Conference on Computer Vision and Pattern Recognition, pages 2843-2851, 2017. 3, 5, 6, 13, 14, 15

[55] Yuxing Tang, Josiah Wang, Boyang Gao, Emmanuel Dellandréa, Robert Gaizauskas, and Liming Chen. Large scale semi-supervised object detection using visual and semantic knowledge transfer. In Proceedings of the IEEE Conference on Computer Vision and Pattern Recognition, pages 21192128, 2016. 2, 3, 4, 5, 6
[56] Sebastian Thrun and Lorien Pratt. Learning to learn. Springer Science \& Business Media, 2012. 2

[57] Jasper Uijlings, Stefan Popov, and Vittorio Ferrari. Revisiting knowledge transfer for training object class detectors. In Proceedings of the IEEE Conference on Computer Vision and Pattern Recognition, pages 1101-1110, 2018. 2, 3

[58] Jasper RR Uijlings, Koen EA Van De Sande, Theo Gevers, and Arnold WM Smeulders. Selective search for object recognition. International journal of computer vision, 104(2):154171, 2013. 3, 14

[59] Oriol Vinyals, Charles Blundell, Timothy Lillicrap, Daan Wierstra, et al. Matching networks for one shot learning. In Advances in neural information processing systems, pages 3630-3638, 2016. 2

[60] Jiajie Wang, Jiangchao Yao, Ya Zhang, and Rui Zhang. Collaborative learning for weakly supervised object detection. arXiv preprint arXiv:1802.03531, 2018. 1, 3, 14

[61] Xin Wang, Thomas E Huang, Trevor Darrell, Joseph E Gonzalez, and Fisher Yu. Frustratingly simple few-shot object detection. ICML, 2020. 2, 7, 8, 9, 13, 14, 15, 17, 18

[62] Yu-Xiong Wang, Deva Ramanan, and Martial Hebert. Metalearning to detect rare objects. In The IEEE International Conference on Computer Vision (ICCV), October 2019. 2, 3, 7, 8, 14

[63] Yuxin Wu, Alexander Kirillov, Francisco Massa, Wan-Yen Lo, and Ross Girshick. Detectron2. https://github. com/facebookresearch/detectron2, 2019. 13

[64] Yongqin Xian, Christoph H Lampert, Bernt Schiele, and Zeynep Akata. Zero-shot learning-a comprehensive evaluation of the good, the bad and the ugly. IEEE transactions on pattern analysis and machine intelligence, 41(9):2251-2265, 2018. 1, 2, 3

[65] Jianxiong Xiao, James Hays, Krista A Ehinger, Aude Oliva, and Antonio Torralba. Sun database: Large-scale scene recognition from abbey to zoo. In 2010 IEEE computer society conference on computer vision and pattern recognition, pages 3485-3492. IEEE, 2010. 6

[66] Xiaopeng Yan, Ziliang Chen, Anni Xu, Xiaoxi Wang, Xiaodan Liang, and Liang Lin. Meta r-cnn: Towards general solver for instance-level low-shot learning. In The IEEE International Conference on Computer Vision (ICCV), October 2019. 2, 3, 7, 8, 13, 14, 15, 17, 18

[67] Hao Yang, Hao Wu, and Hao Chen. Detecting 11k classes: Large scale object detection without fine-grained bounding boxes. In Proceedings of the IEEE International Conference on Computer Vision, pages 9805-9813, 2019. 2, 3

[68] Eloi Zablocki, Patrick Bordes, Benjamin Piwowarski, Laure Soulier, and Patrick Gallinari. Context-aware zero-shot learning for object recognition. arXiv preprint arXiv:1904.12638, 2019. 3

[69] Zhaoyang Zeng, Bei Liu, Jianlong Fu, Hongyang Chao, and Lei Zhang. Wsod2: Learning bottom-up and top-down objectness distillation for weakly-supervised object detection. In Proceedings of the IEEE International Conference on Computer Vision, pages 8292-8300, 2019. 3

[70] Yanzhao Zhou, Yi Zhu, Qixiang Ye, Qiang Qiu, and Jianbin Jiao. Weakly supervised instance segmentation using class 
peak response. In Proceedings of the IEEE Conference on Computer Vision and Pattern Recognition, pages 3791-3800, 2018. 2

[71] Pengkai Zhu, Hanxiao Wang, and Venkatesh Saligrama. Dont even look once: Synthesizing features for zero-shot detection. arXiv preprint arXiv:1911.07933, 2019. 3

[72] C Lawrence Zitnick and Piotr Dollár. Edge boxes: Locating object proposals from edges. In European conference on computer vision, pages 391-405. Springer, 2014. 3 


\section{Appendix}

\section{A. Semi-Supervised Any-shot Segmentation}

In Equation 5 of the main paper we discuss the formulation of the novel segmentation head $f_{\mathbf{W}_{\text {novel }}^{\text {seg }}}(\mathbf{z})$, which is obtained via a structured transfer from the base segmentation head $f_{\mathbf{W}_{\text {base }}^{\text {seg }}}(\mathbf{z})$. We provide more details on how this is implemented in practice. Our implementation of segmentation module can be seen an extension to the Fast R-CNN [19] pipeline described in Section 4 of the main paper. In particular, the segmentation module consists of a transposedconvolution layer ( $\mathrm{nn}$. ConvTranspose 2D), followed by ReLU, and a $1 \times 1$ convolution layer ( $n$ n. Conv2D). The feature vector $\mathbf{z}_{i, j}$ for a proposal $j$ in image $i$ is of dimension $(2048 \times 7 \times 7)$ where 2048 is the number of channels and 7 is the spatial resolution of the proposal's feature map. The segmentation module upsamples $\mathbf{z}$ (as in the main paper we drop $i, j$ indexing) using the transposed convolution layer with a kernel size of 2 , and then produces a class-specific mask using a $1 \times 1$ convolution layer. The resulting mask output is of size $(|\mathcal{C}| \times 14 \times 14)$, where $\mathcal{C}$ is the total number of object classes.

In Eq. 5 of the main paper, $f_{\mathbf{W}_{*}^{\text {seg }}}(\cdot)$ is the class-specific output of the segmentation module obtained after the $1 \times 1$ convolution. During training, we use the same loss formulation for $\mathcal{L}_{\text {mask }}$ as described in [20], where a per-pixel binary cross-entropy loss is used. During inference, the mask is interpolated to fit the regressed proposal (as obtained by Eq.(2) and Eq.(4) in the main paper) to produce the final output. For the semi-supervised zero-shot $(k=0)$ scenario, the predictions for novel classes can be done as in Eq. (5) but omitting the "instance-level direct adaptation" term.

\section{B. Implementation Details}

For base-training, we train our model jointly with weak-supervision and base-detection/-segmentation losses with equal weighting (see Section 4.3). In particular, we use image-level data for all the classes to train the weakly-supervised OICR [54] branch, and use detection/segmentation data of base classes for training base detectors/segmentors. Unless pretrained proposals are used, the proposals used for training weakly-supervised branch come from the RPN trained using the base-detection branch. For the zero-shot experiments $(k=0)$ in Section 5.1, similar to the baselines, we replace the RPN with the precomputed MCG proposals [38]. We use 4 Nvidia Tesla T4 GPUs to train models. We build on top of Detectron2 [63] library written in PyTorch [36] framework, and unless mentioned, we keep their default configuration: SGD as the optimizer, RoI Align [20] as the pooling method, ResNet layer sizes/parameters. We use the standard loss for Faster $\mathrm{R}-\mathrm{CNN}$, i.e., cross-entropy loss for classifier and smooth-L1 loss for regressor as described in [42].
Note that, in the following text, an iteration refers to a gradient step, and not the total number of examples in the training set.

Semi-Supervised Object Detection We train on the MSCOCO 2015 [32] data for semi-supervised zero-shot $(k=0)$ and MSCOCO 2017 [32] for semi-supervised fewshot $(k>0)$ experiments. We use $270 \mathrm{~K}$ iterations (default in Detectron2 [63]) to account for more data. For fine-tuning, we use 1000 iterations for 12-shot, 3000 iterations for 33shot, 6000 iterations for 55-shot, 8000 iterations for 76 -shot, and 10000 iterations for 96 -shot experiments.

Few-shot Object Detection: VOC. We train on VOC $07+$ 12 dataset. We use a learning rate of 0.02 over $30 \mathrm{~K}$ iterations. We decrease the learning rate by a factor of 10 at $12 \mathrm{~K}$ and $24 \mathrm{~K}$ iteration.

For fine-tuning, we are given $k$-shot data for novel classes where $k \in\{1,2,3,5,10\}$. We linearly scale the number of SGD steps for optimizing over the $k$-shot data. In particular, we choose 50 iterations for $k=1,100$ iterations for $k=2$, and similarly linearly scale to 500 iterations for $k=10$.

Few-shot Object Detection: COCO. In the case of COCO dataset, we use $270 \mathrm{~K}$ iterations (default in Detectron2 [63]) to account for more data as compared to VOC. For fine-tuning, we use 500 iterations for 10 -shot and 1500 iterations for 30-shot experiment.

Weakly-supervised Object Detection The results are shown in Section E. Here we use a pre-trained VGG-16 [47] as the backbone to be consistent with the prior state-ofthe-art works [5, 54, 2, 43]. We use a learning rate of 0.001 over $40 \mathrm{~K}$ iterations for optimization, dropping it down to 0.0001 for the next $30 \mathrm{~K}$ iterations.

We will also make all our code publicly available.

\section{Few-shot Object Detection on MSCOCO}

As described in Section 5.2 of the main paper, we compare the performance of UniT against state-of-the-art approaches on the task of Few-Shot Object Detection on the MSCOCO dataset [32]. Please refer to Section 5.2 for task and dataset specifications. Similar to [61, 66], we choose ResNet-50 [21] as the backbone. The k-shot tasks are sampled following [66] with $k \in\{0,10,30\}$. Due to lack of space, results in Table 4 of the main paper only compared our model against the baselines on 10-shots. Here we present the complete comparison in Table A1.

As can be seen from Table A1 we get a significant boost in performance even on large number of shots $k=30$. As UniT uses additional weak image-level data for novel classes, this is not an equivalent comparison (see Sec. 5.3 of the main paper for comparisons under similar annotation budget). But 


\begin{tabular}{|c|c|c|c|c|c|c|c|c|c|c|c|c|c|}
\hline \#Shots & & AP & $\mathrm{AP}_{50}$ & $\mathrm{AP}_{75}$ & $\mathrm{AP}_{S}$ & $\mathrm{AP}_{M}$ & $\mathrm{AP}_{L}$ & $\mathrm{AR}_{1}$ & $\mathrm{AR}_{10}$ & $\mathrm{AR}_{100}$ & $\mathrm{AR}_{S}$ & $\mathrm{AR}_{M}$ & $\mathrm{AR}_{L}$ \\
\hline$k=0$ & UniT (Ours) & 18.9 & 36.1 & 17.5 & 8.7 & 20.4 & 27.6 & 19.1 & 33.3 & 35.0 & 16.5 & 35.5 & 48.5 \\
\hline \multirow{6}{*}{$k=10$} & Transfer: FRCN [66] & 6.5 & 13.4 & 5.9 & 1.8 & 5.3 & 11.3 & 12.6 & 17.7 & 17.8 & 6.5 & 14.4 & 28.6 \\
\hline & Kang et al. [24] & 5.6 & 12.3 & 4.6 & 0.9 & 3.5 & 10.5 & 10.1 & 14.3 & 14.4 & 1.5 & 8.4 & 28.2 \\
\hline & Wang et al. [62] & 7.1 & 14.6 & 6.1 & 1.0 & 4.1 & 12.2 & 11.9 & 15.1 & 15.5 & 1.7 & 9.7 & 30.1 \\
\hline & Yan et al. [66] & 8.7 & 19.1 & 6.6 & 2.3 & 7.7 & 14.0 & 12.6 & 17.8 & 17.9 & 7.8 & 15.6 & 27.2 \\
\hline & Wang et al. [61] & 10.0 & - & 9.3 & - & - & - & - & - & - & - & - & - \\
\hline & UniT (Ours) & 21.7 & 40.8 & 20.6 & 9.1 & 23.8 & 31.3 & 21.1 & 35.1 & 36.4 & 16.5 & 37.5 & 51.0 \\
\hline \multirow{6}{*}{$k=30$} & Transfer: FRCN [66] & 11.1 & 21.6 & 10.3 & 2.9 & 8.8 & 18.9 & 15.0 & 21.1 & 21.3 & 10.1 & 17.9 & 33.2 \\
\hline & Kang et al. [24] & 9.1 & 19.0 & 7.6 & 0.8 & 4.9 & 16.8 & 13.2 & 17.7 & 17.8 & 1.5 & 10.4 & 33.5 \\
\hline & Wang et al. [62] & 11.3 & 21.7 & 8.1 & 1.1 & 6.2 & 17.3 & 14.5 & 18.9 & 19.2 & 1.8 & 11.1 & 34.4 \\
\hline & Yanet al. [66] & 12.4 & 25.3 & 10.8 & 2.8 & 11.6 & 19.0 & 15.0 & 21.4 & 21.7 & 8.6 & 20.0 & 32.1 \\
\hline & Wang et al. [61] & 13.7 & - & 13.4 & - & - & - & - & - & - & - & - & - \\
\hline & UniT (Ours) & 23.1 & 43.0 & 21.6 & 9.8 & 25.3 & 33.8 & 22.4 & 36.7 & 37.9 & 16.5 & 38.7 & 53.3 \\
\hline
\end{tabular}

Table A1: Complete few-shot object detection results on COCO. FRCN=Faster R-CNN with ResNet-50 [21] backbone. Similar to $[61,66]$, we use ResNet-50 as the backbone.

we want to highlight that such data is readily available, much cheaper to obtain [4], and leads to a significant improvement in performance.

\section{Comparison to Few-shot Instance Segmen- tation}

As described in Section 5.2 of the main paper, we analyse the performance of our proposed approach on the task of Few-shot Instance Segmentation. Please refer to Section 5.2 for task and dataset specifications. Similar to [66], we choose ResNet-50 [21] as the backbone and use an additional segmentation head (as described in Section A of the supplementary). The $k$-shot tasks are sampled following [66] with $k \in\{0,5,10,20\}$, and we follow the standard evaluation metrics on COCO [20]. The complete results are shown in Table A2. Our approach consistently improves over [66], demonstrating that our approach is not limited to bounding boxes and is able to generalize over the type of downstream structured label, including segmentation mask.

\section{E. Weakly-Supervised Object Detection}

Dataset. For completeness, we compare our approach against existing weakly-supervised object detection approaches. We evaluate our approach on VOC 2007 [14] which consists of a trainval set of 5011 images for training and 4951 images for test, keeping in line with the prior related works [5, 54, 53, 60, 2]. As we assume instance-level supervision for base classes in the dataset, we report performance on the novel classes for three different splits specified in Section 5.2 and $[61,66]$. Note that, similar to the baselines, we assume zero instance-level supervision for novel classes (i.e. $k=0$ ).

Results. Table A3 provides a summary of the results. Similar to [5, 54, 53, 2, 43], we use a pre-trained proposal network (Selective Search [58]) and an ImageNet pretrained VGG-16 [47] backbone for fair comparison. As we assume additional supervision for base classes, this is not an equivalent comparison. However, for novel classes, all methods have access to the same data. Our results beat the strong baseline of [2] on 2 out of 3 novel splits, and provides comparable performance to the state-of-the-art [43]. Our significant improvement over OICR [54], which we build upon, on novel classes ( $~ 35 \%$ on average across three splits) highlights the effectiveness of our proposed transfer. We note that our approach is agnostic to the model architecture used for weak-supervision, and the model performance can be improved further if built on top of better weak detectors (e.g. [2, 43]).

\section{F. Explanation of Annotation Budget Conver- sion Factor}

In Section 5.3 of the main paper, we perform a constraint annotation budget analysis to facilitate an equivalent comparison to existing few-shot detection works [61,24], while simultaneously analysing the relative importance of imagelevel annotations. To this end, for each value of $k$ instancelevel annotations in the few-shot setup, we trained a variant of UniT that assumes only $7 \times k$ image-level annotations for novel classes, which is referred to as $\mathrm{UniT}_{\text {budget }=k}$. Here we describe the rationale behind using this conversion factor of 7 for the VOC dataset [13], derived from annotation times reported in the literature.

Image-Level Annotation (20.0 sec/image). As per [4] and [34], collecting image-level labels takes 1 second per class. As VOC [13] has 20 object classes, generating an imagelevel annotation is expected to take 20 seconds per image [4].

Instance-Level Bounding Box Annotation (137.2 sec/image). [4] reports an expected annotation time of 239.7 seconds per image for VOC [13]. However, this estimate additionally assumes time required to obtain 


\begin{tabular}{|c|c|c|c|c|c|c|c|c|c|c|c|c|c|}
\hline \multirow[b]{2}{*}{ \#Shots } & \multirow[b]{2}{*}{ Method } & \multicolumn{6}{|c|}{ Box } & \multicolumn{6}{|c|}{ Mask } \\
\hline & & AP & $\mathrm{AP}_{50}$ & $\mathrm{AP}_{75}$ & $\mathrm{AP}_{S}$ & $\mathrm{AP}_{M}$ & $\mathrm{AP}_{L}$ & $\mathrm{AP}$ & $\mathrm{AP}_{50}$ & $\mathrm{AP}_{75}$ & $\mathrm{AP}_{S}$ & $\mathrm{AP}_{M}$ & $\mathrm{AP}_{L}$ \\
\hline$k=0$ & Ours & 20.2 & 36.8 & 19.5 & 8.5 & 20.9 & 28.9 & 17.6 & 32.7 & 17.0 & 5.6 & 17.6 & 27.7 \\
\hline \multirow{2}{*}{$k=5$} & Yan et al. & 3.5 & 9.9 & 1.2 & 1.2 & 3.9 & 5.8 & 2.8 & 6.9 & 1.7 & 0.3 & 2.3 & 4.7 \\
\hline & Ours & 22.1 & 39.9 & 21.7 & 9.2 & 23.0 & 31.7 & 20.0 & 37.5 & 19.0 & 6.1 & 19.4 & 31.1 \\
\hline \multirow{2}{*}{$k=10$} & Yan et al. [66] & 5.6 & 14.2 & 3.0 & 2.0 & 6.6 & & 4.4 & 10.6 & 3.3 & 0.5 & 3.6 & 7.2 \\
\hline & Ours & 22.8 & 41.6 & 21.9 & 9.4 & 24.4 & 32.3 & 20.5 & 38.6 & 19.7 & 6.0 & 20.5 & 31.8 \\
\hline \multirow{2}{*}{$k=20$} & Yan et al. [ & 6.2 & 16.6 & 2.5 & 1.7 & 6.7 & 9.6 & 6.4 & 14.8 & 4.4 & 0.7 & 4.9 & 9.3 \\
\hline & Ours & 23.8 & 42.7 & 23.8 & 9.9 & 24.9 & 34.6 & 21.4 & 39.2 & 21.2 & 6.2 & 20.5 & 33.2 \\
\hline
\end{tabular}

Table A2: Complete Table for Few-shot Instance Segmentation on COCO. All models use ImageNet [11] pretrained ResNet-50 [21] as the backbone.

\begin{tabular}{cccccc|ccccccccccccccc}
\hline & \multicolumn{4}{c}{ Novel split 1 } & \multicolumn{4}{c}{ Novel split 2 } & \multicolumn{4}{c}{ Novel split 3 } \\
Method & bird & bus & cow & mbike & sofa & mean & aero & bottle & cow & horse & sofa & mean & boat & cat & mbike & sheep & sofa & mean \\
\hline WSDDN [5] & 31.5 & 64.5 & 35.7 & 55.6 & 40.7 & 45.6 & 39.4 & 12.6 & 35.7 & 34.4 & 40.7 & 32.6 & 16.3 & 42.6 & 55.6 & 30.2 & 40.7 & 37.1 \\
OICR [54] & 31.1 & 65.1 & 44.7 & 65.5 & 46.9 & 50.7 & 58.0 & 13.0 & 44.7 & 37.8 & 46.9 & 40.1 & 19.4 & 28.4 & 65.5 & 41.7 & 46.9 & 40.4 \\
PCL [53] & 39.3 & 62.9 & 52.5 & 67.7 & 57.5 & 56.0 & 54.4 & 15.7 & 52.5 & 39.3 & 57.5 & 43.9 & 19.2 & 30.0 & 67.7 & 46.6 & 57.5 & 44.2 \\
PredNet [2] & 52.8 & $\mathbf{7 4 . 5}$ & 53.0 & 70.8 & $\mathbf{6 0 . 7}$ & 62.4 & 66.7 & 24.7 & 53.0 & $\mathbf{6 9 . 9}$ & $\mathbf{6 0 . 7}$ & 55.0 & $\mathbf{3 1 . 4}$ & 67.3 & 70.8 & 54.6 & $\mathbf{6 0 . 7}$ & 57.0 \\
Wetectron [43] & $\mathbf{5 7 . 0}$ & 69.1 & 73.2 & $\mathbf{7 7 . 7}$ & 53.8 & $\mathbf{6 6 . 2}$ & $\mathbf{6 8 . 8}$ & $\mathbf{2 8 . 9}$ & 73.2 & 54.4 & 53.8 & $\mathbf{5 5 . 8}$ & 27.7 & 67.0 & $\mathbf{7 7 . 7}$ & $\mathbf{6 4 . 1}$ & 53.8 & 58.1 \\
\hline UniT + OICR (Ours) & 45.5 & 71.8 & $\mathbf{7 5 . 1}$ & 74.0 & 52.7 & 63.8 & 64.0 & 17.6 & $\mathbf{7 3 . 8}$ & 59.9 & 54.4 & 53.9 & 30.8 & $\mathbf{7 1 . 7}$ & 74.9 & 63.4 & 55.1 & $\mathbf{5 9 . 2}$ \\
\hline
\end{tabular}

Table A3: Comparison to Weakly-supervised methods. Comparison on the three novel splits described in Section 5.2 and [66, 61]. All methods use VGG-16 [47] as the backbone.

instance segmentations. As the methods in the few-shot detection domain $[61,24]$ that we compare against in Section 5.3 only use bounding box annotations to train their models, we modify this estimate of 239.7 seconds to get a more accurate conversion factor.

There are 1.5 object classes per image on average in the VOC 2012 dataset [13]. As mentioned in [4], it takes 1 second to annotate every object class that is not present (i.e. obtain an image-level "no object" label). This amounts to $20-1.5=18.5$ seconds of labeling time. In addition, there are 2.8 object instances on average in the VOC dataset [13]. As mentioned in [50], if we use their efficient system to crowdsource annotations, the median time to get a bounding box annotation is 42.4 seconds (note that the average time is much higher at 88.0 seconds). Therefore, it is expected to take $2.8 \times 42.4=118.7$ seconds to obtain bounding box annotations per image in VOC. Thus, the total annotation time is: $18.5+118.7=137.2$ seconds per image.

It can be seen that obtaining instance-level annotations is approximately $137.2 / 20 \approx 7$ times more time consuming. Hence, we use a conversion factor of 7 for our experiments in Section 5.3. Also, according to [35], this estimate of 42.4 seconds per bounding box is conservative. It doesn't take into account the errors encountered during crowdsourcing (e.g. some boxes being rejected and needing to be redrawn). Therefore, we expect this conversion factor of 7 to be higher in practice.

\section{G. Ablation study}

Please refer to Section 5.2 of the main paper for a detailed explanation of task setup. We perform ablation over the terms used in Equations (3), (4), and (5) of the main paper on the novel classes for the VOC [32] and MSCOCO [32] datasets. We show the benefit of using our proposed transfer both in the zero-shot $(k=0)$ and the few-shot $(k>0)$ setting. We first describe the ablated variants of our model, and then discuss the results. The results are summarized in Table A4 for VOC and Table A5 for MSCOCO.

We start by forming detectors using only the weaklysupervised branch $f_{\mathbf{W}_{\text {weak }}}$ (denoted as "weak" in Tables A4 and A5), and progressively add refinement terms to observe their impact on detection/segmentation performance. We then incorporate the transfer from the base classes $f_{\Delta \mathbf{W}_{b a s e}^{c l s}}$ into the weak detector (see Equation 3 in the main paper). For each novel class, we first compare to a simple baseline approach: averaging over all the base classes (denoted by weak $+\operatorname{avg}(\Delta)$ ). We note that a naïve averaging doesn't provide any performance improvements, suggesting the need for a more informed transfer between base and novel classes. We then explore the role of proposed similarity matrices, detailed in Section 4.2 of the main paper. The similarity matrix between base and novel classes can be decomposed into two components: lingual similarity $\mathbf{S}^{l i n}$ and visual similarity $\mathbf{S}^{v i s}(\mathbf{z})$. We analyse the impact of using the aforementioned similarities in obtaining category-aware classifiers, regres- 


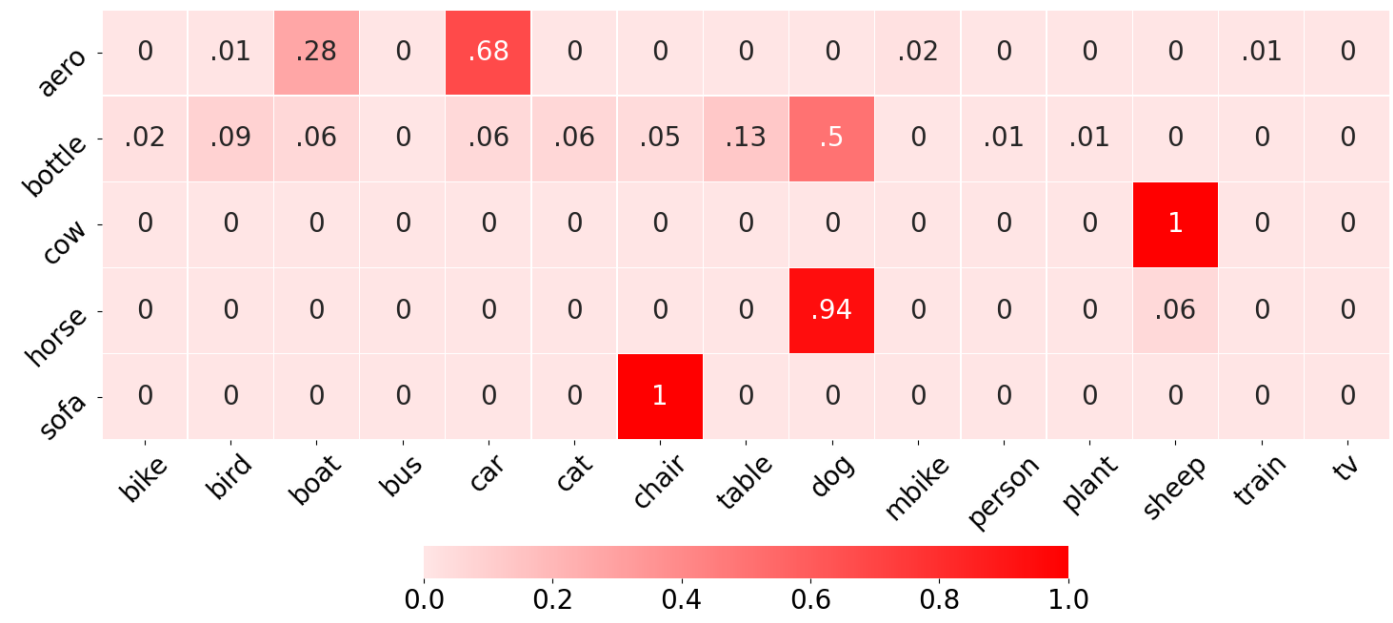

Figure A1: Normalized lingual similarity matrix for the second novel split in PASCAL VOC. Note that $\mathbf{S}^{\text {lin }}$ is proposalagnostic. Most of the similarities are intuitive and semantic - sofa is most similar to a chair; horse to a dog and a sheep; cow is similar to a sheep; aeroplane is related to other transportation vehicles like car and boat. A notable departure is a bott le which has no closely related categories among base classes, resulting in less interpretable similarity and transfer.

\begin{tabular}{|c|c|c|c|c|c|c|c|c|c|c|c|c|}
\hline \multirow[t]{2}{*}{ Method } & \multicolumn{6}{|c|}{$k=0$} & \multicolumn{6}{|c|}{$k=5$} \\
\hline & bird & bus & cow & mbike & sofa & mean & bird & bus & cow & mbike & sofa & mean \\
\hline weak & 58.1 & 73.5 & 70.4 & 68.5 & 49.1 & 63.9 & 59.6 & 75.9 & 72.7 & 71.8 & 55.1 & 67.0 \\
\hline weak $+\operatorname{avg}(\Delta)$ & 57.8 & 73.5 & 71.2 & 68.3 & 47.8 & 63.7 & 60.0 & 76.0 & 73.6 & 71.2 & 54.2 & 67.0 \\
\hline weak $+\mathbf{S}_{c l s}^{\text {lin }}$ & 55.0 & 74.2 & 73.6 & 70.8 & 46.6 & 64.1 & 58.3 & 76.8 & 76.1 & 73.5 & 52.1 & 67.4 \\
\hline weak $+\mathbf{S}_{c l s}^{l i n}+\mathbf{S}_{c l s}^{v i s}$ & 56.2 & 74.6 & 73.9 & 71.2 & 48.8 & 64.9 & 59.3 & 77.1 & 77.2 & 73.9 & 52.0 & 67.9 \\
\hline weak $+\mathbf{S}_{c l s, r e g}^{l i n}+\mathbf{S}_{c l s, r e g}^{v i s}$ & 69.9 & 83.4 & 86.1 & 81.1 & 57.8 & 75.7 & 69.8 & 84.0 & 86.2 & 81.3 & $\mathbf{5 9 . 0}$ & 76.1 \\
\hline
\end{tabular}

Table A4: Ablation study on the VOC $07+12$ dataset. Please refer to Section $\mathrm{G}$ for model definitions. We report $\mathrm{AP}_{50}$ on the first split in [24], and show results on zero-shot $(k=0)$ and few-shot $(k=5)$.

sors, and segmentors. Following the terms in Eq. (3), (4), and (5) of the main paper, we define ablated variants of our final model.

- "weak $+\mathbf{S}_{c l s}^{\text {lin }}$ " is the model where-in the categoryaware classifier for the novel classes is obtained by using only the lingual similarity, and the category-aware regressor is fixed to predict zeros (i.e. the model uses the output of the category-agnostic Fast-RCNN regressor rbox). As there is no estimate for the novel mask head, we predict a uniform mask over the selected bounding box region.

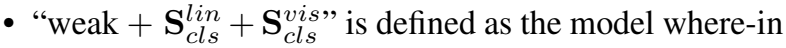
the category-aware classifier for the novel classes is obtained by using both lingual and visual similarities (Eq. (3)), and the category-aware regressor is fixed to predict zeros. As there is no estimate for the novel mask head, we predict a uniform mask over the selected bounding box region.
- "weak $+\mathbf{S}_{c l s, r e g}^{l i n}+\mathbf{S}_{c l s, r e g}^{v i s}$ " is defined as the model where-in both the category-aware classifier and the category-aware regressor for the novel classes is obtained by using lingual and visual similarities (Eq. (3)) and (4)). As there is no estimate for the novel mask head, we predict a uniform mask over the selected bounding box region. For experiments in Table A4, this is the complete UniT model.

- Finally, "weak $+\mathbf{S}_{c l s, r e g, s e g}^{l i n}+\mathbf{S}_{c l s, r e g, s e g}^{v i s}$ " is defined as the model where-in the category-aware classifier, the category-aware regressor, and the category-aware segmentor for the novel classes is obtained by using lingual and visual similarities (Eq. (3), (4), and (5)). For experiments in Table A5, this is the complete UniT model.

From Table A4 it can be seen that adding each of our terms improves model performance. Particularly, transferring information from base regressors to novel regressors 


\begin{tabular}{|c|c|c|c|c|c|c|c|c|c|c|c|c|}
\hline \multirow[b]{2}{*}{ Method } & \multicolumn{6}{|c|}{ Box } & \multicolumn{6}{|c|}{ Mask } \\
\hline & AP & $\mathrm{AP}_{50}$ & $\mathrm{AP}_{75}$ & $\mathrm{AP}_{S}$ & $\mathrm{AP}_{M}$ & $\mathrm{AP}_{L}$ & AP & $\mathrm{AP}_{50}$ & $\mathrm{AP}_{75}$ & $\mathrm{AP}_{S}$ & $\mathrm{AP}_{M}$ & $\mathrm{AP}_{L}$ \\
\hline weak & 10.7 & 28.3 & 5.1 & 5.3 & 12.9 & 14.3 & 4.7 & 17.6 & 1.1 & 2.1 & 6.0 & 6.0 \\
\hline weak $+\operatorname{avg}(\Delta)$ & 10.7 & 28.3 & 5.1 & 5.3 & 12.9 & 14.2 & 4.7 & 17.5 & 1.1 & 2.0 & 6.0 & 6.0 \\
\hline weak $+\mathbf{S}_{c l s}^{l i n}$ & 11.4 & 29.7 & 5.7 & 6.3 & 13.2 & 14.6 & 5.0 & 18.5 & 1.0 & 2.3 & 6.0 & 6.1 \\
\hline weak $+\mathbf{S}_{c l s}^{l i n}+\mathbf{S}_{c l s}^{v i s}$ & 11.7 & 29.9 & 6.0 & 6.4 & 13.3 & 14.7 & 5.2 & 18.7 & 1.2 & 2.4 & 6.1 & 6.1 \\
\hline weak $+\mathbf{S}_{c l s, r e q}^{l i n}+\mathbf{S}_{c l s, r e q}^{v i s}$ & 20.2 & 36.8 & 19.5 & 8.5 & 20.9 & 28.9 & 8.5 & 25.0 & 4.5 & 2.8 & 8.7 & 12.0 \\
\hline weak $+\mathbf{S}_{c l s, r e g, s e g}^{l i n}+\mathbf{S}_{c l s, r e g, s e g}^{v i s}$ & 20.2 & 36.8 & 19.5 & 8.5 & 20.9 & 28.9 & $\mathbf{1 7 . 6}$ & 32.7 & 17.0 & 5.6 & 17.7 & 27.7 \\
\hline
\end{tabular}

Table A5: Ablation study on the MSCOCO dataset. Please refer to Section $\mathrm{G}$ for model definitions. The results show the zero shot $(k=0)$ performance of the models.

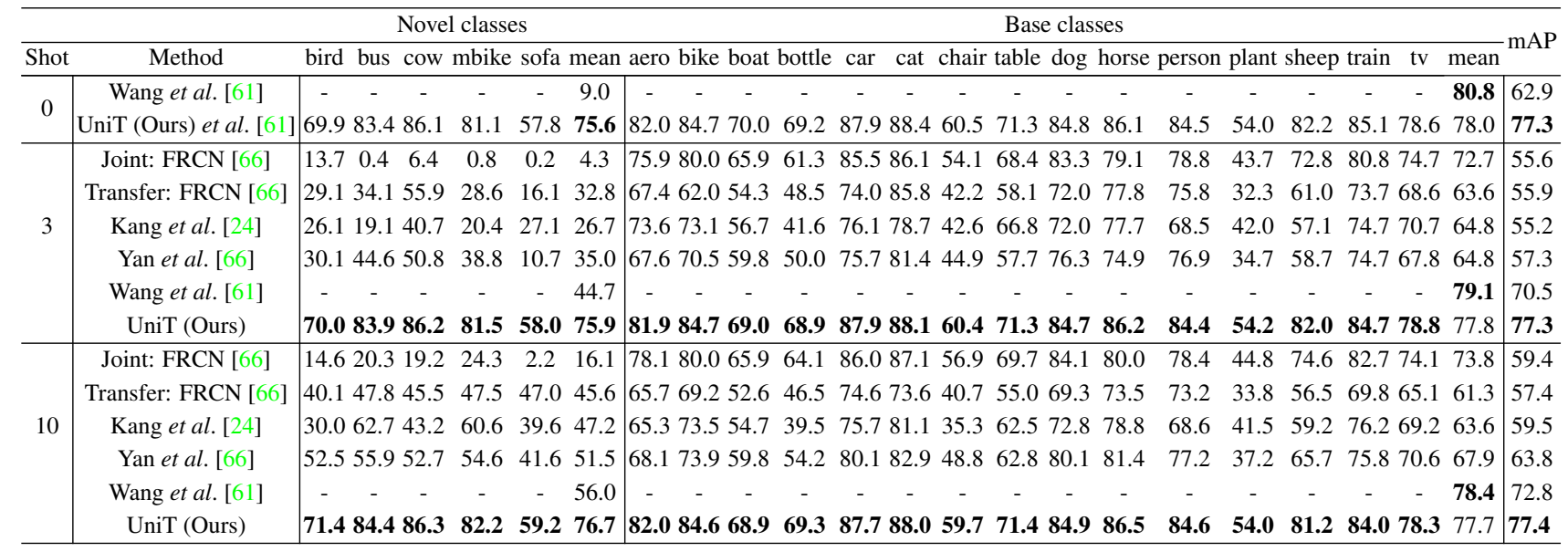

Table A6: Base class performance on VOC. AP and mAP on VOC2007 test set for novel classes and base classes of the first base/novel split. We evaluate the performance for 0/3/10-shot novel-class examples with FRCN under ResNet-101. Note that as Wang et al. [61] do not report per-class performance numbers, we just show their reported aggregated performance. Additionally, all models use the same amount of annotation for the base classes.

("weak $+\mathbf{S}_{c l s, r e g}^{l i n}+\mathbf{S}_{c l s, r e g}^{\text {vis }}$ ") provides a significant boost. We additionally show that this trend holds even when the models are fine-tuned on few-shot $(k=5)$ examples for novel classes. Table A5 further highlights the effectiveness of our proposed transfer on segmentation masks ("weak $+\mathbf{S}_{\text {cls,reg,seg }}^{\text {lin }}+\mathbf{S}_{\text {cls,reg,seg }}^{\text {vis }}$ "). Note that the final two lines in Table A5 only differ in mask AP performance as the regression transfer terms are identical in both the ablated models.

Figure A3 provides qualitative examples to further highlight the impact of using our proposed transfer from base to novel classes. Column (a) in Figure A3 refers to "weak", column (b) refers to "weak $+\mathbf{S}_{c l s}^{l i n}+\mathbf{S}_{c l s}^{v i s ",}$, column (c) refers to the "weak $+\mathbf{S}_{c l s, r e g}^{l i n}+\mathbf{S}_{c l s, r e g}^{\text {vis }}$ "with $k=0$, and column (d) refers to the "weak $+\mathbf{S}_{c l s, r e g}^{l i n}+\mathbf{S}_{c l s, r e g}^{v i s}$ " after being trained with $k=5$ shots. It can be seen that the "weak" model either fails to identify all objects or doesn't generate high-probability proposals for the desired objects (column

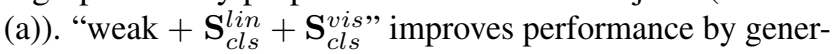
ating a bunch of reasonable proposals (column (b)). "weak
$+\mathbf{S}_{c l s, r e g}^{l i n}+\mathbf{S}_{c l s, r e g}^{v i s}$ " further refines the proposals to obtain accurate bounding boxes for the objects (column (c)). Finally, fine-tuning on $k=5$ shots improves the bounding box confidence and slightly refines the predictions (column (d)).

\section{H. Analysis of Similarity Matrices}

As described in Section 4 of the main paper, the key contribution of our approach is the ability to semantically decompose the classifiers, detectors and segmentors of novel classes into their base classes' counterparts. To this end, we define a proposal-aware similarity $\mathbf{S}(\mathbf{z}) \in \mathbb{R}^{\left|C_{\text {novel }}\right| \times\left|C_{\text {base }}\right|}$, which is further decomposed into two components: lingual $\mathbf{S}^{\text {lin }}$ and visual $\mathbf{S}^{\text {vis }}(\mathbf{z})$ similarity. Please refer to Section 4.2 of the main paper on details pertaining to how these similarities are computed.

We qualitatively visualize these similarity matrices to highlight the intuitive semantics learned by our proposed model. Figure A1 shows the normalized lingual similarity matrix $\mathbf{S}^{l i n} \in \mathbb{R}^{\left|C_{\text {novel }}\right| \times\left|C_{\text {base }}\right|}$ for the second novel split in PASCAL VOC. Figure A2 shows the normalized visual sim- 
ilarity $\mathbf{S}^{v i s}(\mathbf{z}) \in \mathbb{R}^{\left|C_{\text {base }}\right|}$ for each proposal $\mathbf{z}$ (highlighted in blue).

\section{Few-shot Performance on VOC's Base Classes}

Due to lack of space, in the main paper, we focus on the detection/segmentation results obtained on the novel object classes; however, our model also learns to detect/segment base class objects as well. We now illustrate that our proposed method improves performance on novel classes as the value of $k$ is increased, without any significant performance drop on base classes. The experimental setup and baselines are identical to the one described in Section 5.2 of the main paper. Table A6 summarizes results on VOC [14] for the 1 -st novel split with $k$-shots, $k \in\{0,3,10\}$.

It is important to note that we are not using any additional annotations for the base classes (when compared to $[24,66,61])$. It can be seen that our model's performance on novel classes steadily increases with increased $k$, while simultaneously retaining accuracy on base classes.

Our slightly poorer performance on base classes compared to [61] can be attributed to the fact that [61] use feature pyramid networks (FPN) [31] whereas we don't. According to [31], FPN provides a performance improvement of about 3.8 on $\mathrm{AP}_{50}$ (See Table 3 in [31], rows (a) and (c)). Our approach, despite not using FPNs, only performs 2.8 points poorer on $k=0$. In addition, this gap reduces as the value of $k$ is increased (1.3 $\mathrm{AP}_{50}$ on $k=3 ; 0.7 \mathrm{AP}_{50}$ on $k=10$ ). Also, compared to [61], UniT has a smaller drop in base class performance as $k$ is increased. As an example, when $k$ is increased from 0 to 5 , UniT has a performance drop of $0.2 \mathrm{AP}_{50}$ on base classes whereas [61] has a larger drop of $1.7 \mathrm{AP}_{50}$. This observation highlights the fact that our proposed approach is better at retaining base class information compared to the existing state-of-the-art in [61]. This improved retention can be mainly attributed to the structured decomposition of our detectors: weak detector + learned refinement. We believe that such a decomposition results in an inductive model bias that leads to convergence to an ultimately better solution.

\section{J. Additional Visualizations on MSCOCO De- tection and Segmentation}

We show additional visualizations highlighting the performance of our approach on the MSCOCO [32] dataset. The experimental setup is identical to the ones described in Sections 5.2 of the main paper. Figure A4 shows additional examples for the task of object detection, and Figure A5 shows additional examples for the task of instance segmentation. Note that these visualizations are generated on novel classes under the $k=0$ setup. 

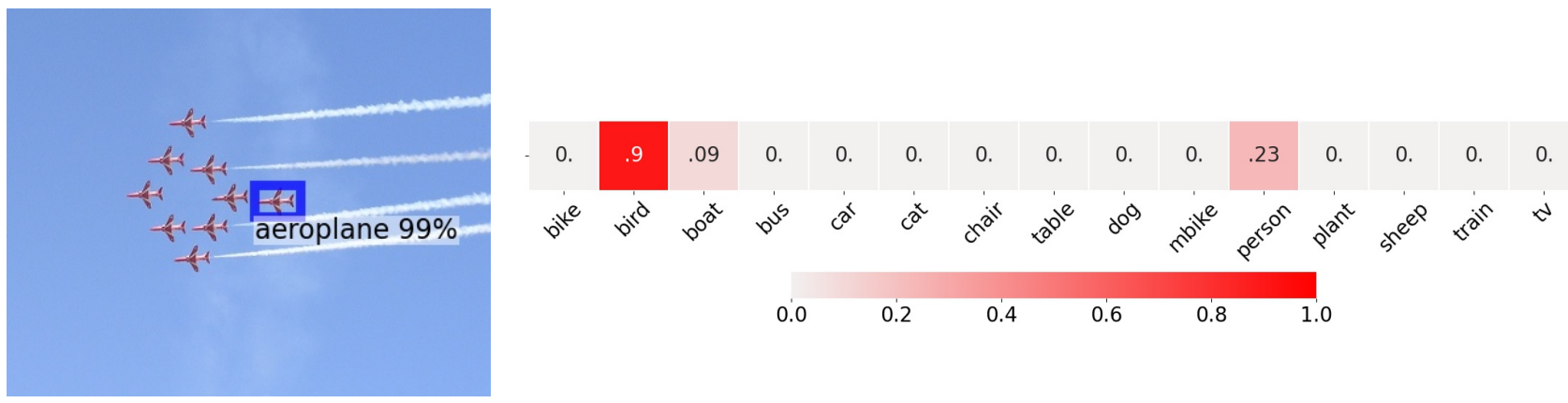

(a) Complementary to $\mathbf{S}^{\text {lin }}$ that assigns weights to classes boat and car, $\mathbf{S}^{\text {vis }}(\mathbf{z})$ is additionally able to capture that an aeroplane flying in the sky shares some visual characteristics with the class bird.
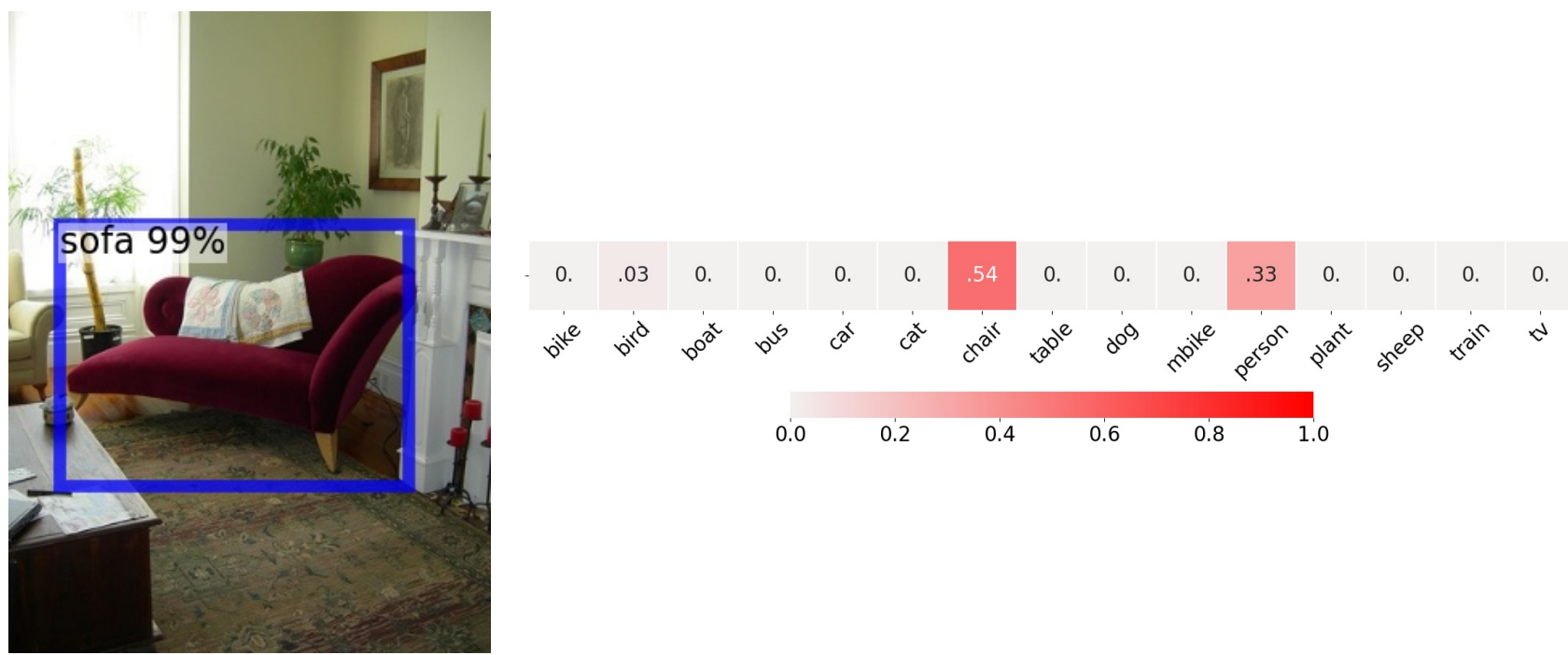

(b) Complementary to $\mathbf{S}^{\text {lin }}$ that gives a large weight to the class chair, $\mathbf{S}^{\text {vis }}(\mathbf{z})$ is additionally able to capture that there is a high correlation between the class person and the class sofa. This follows the common observation that people are likely to be sitting on a sofa.
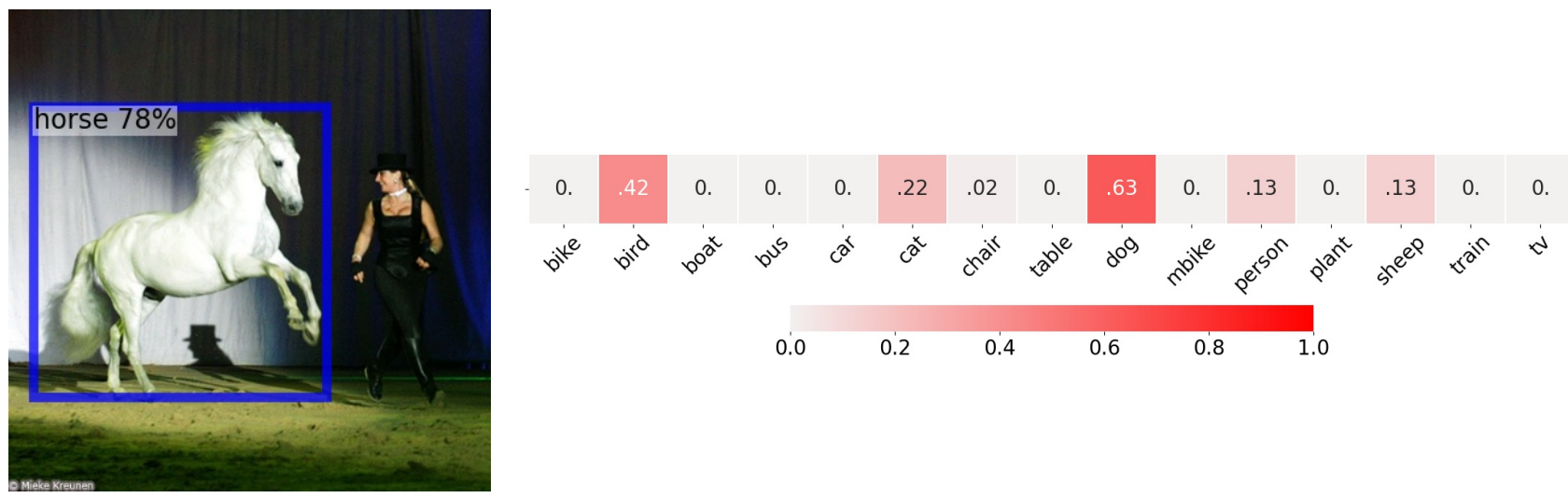

(c) Complementary to $\mathbf{S}^{\text {lin }}$ that gives a large weight to the class dog, $\mathbf{S}^{\text {vis }}(\mathbf{z})$ is additionally able to capture that the class horse is visually similar to other animal classes bird, cat, and sheep. Additionally, it captures a correlation with the class person, which follows from the observation that humans usually ride horses.

Figure A2: Visual similarity for the second novel split in PASCAL VOC. The input proposal $\mathrm{z}$ is highlighted in blue. The visual similarity captures complementary information to the lingual similarity. 


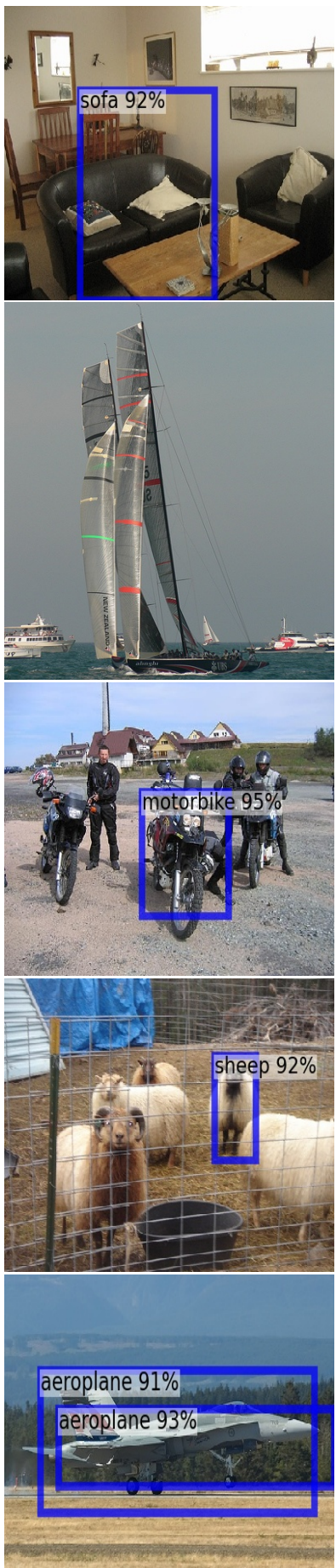

(a)
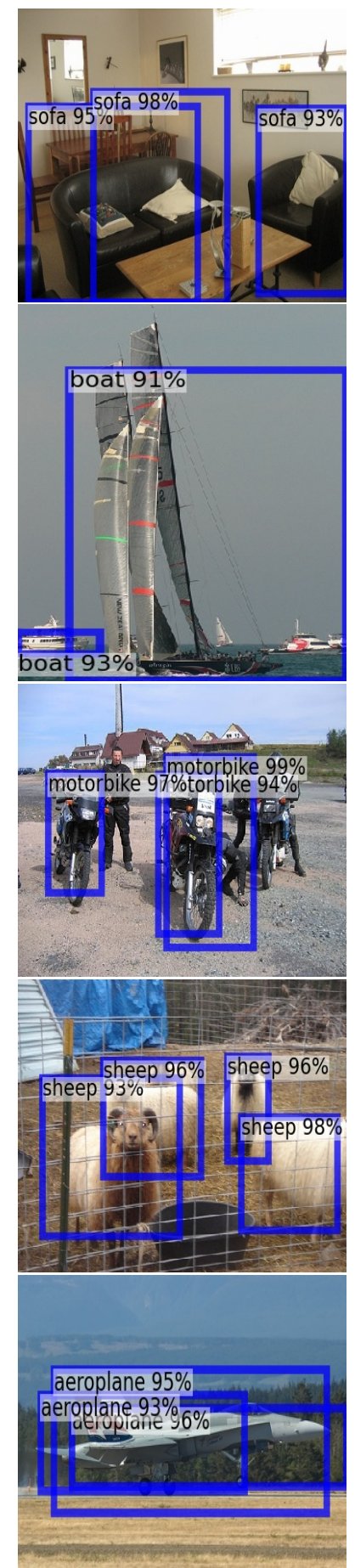

(b)
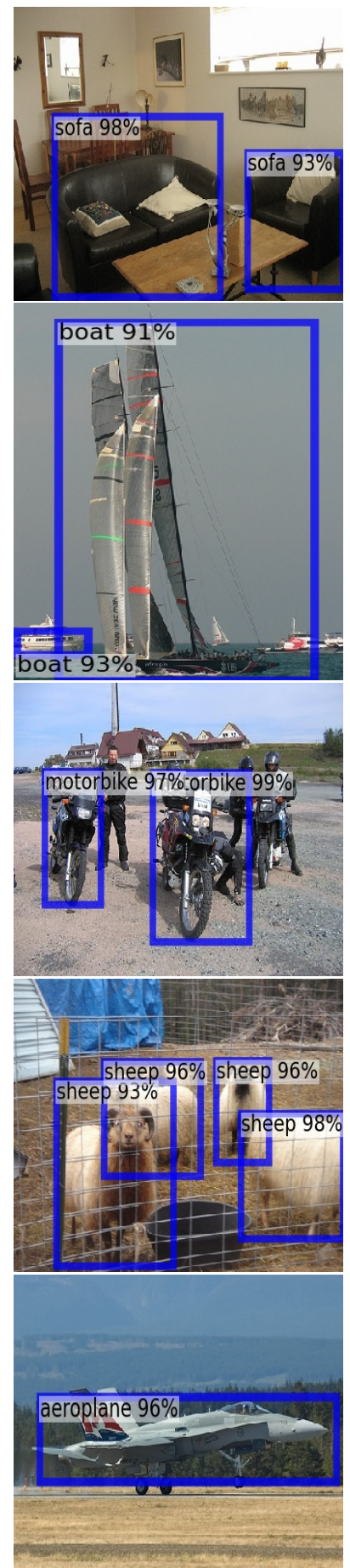

(c)
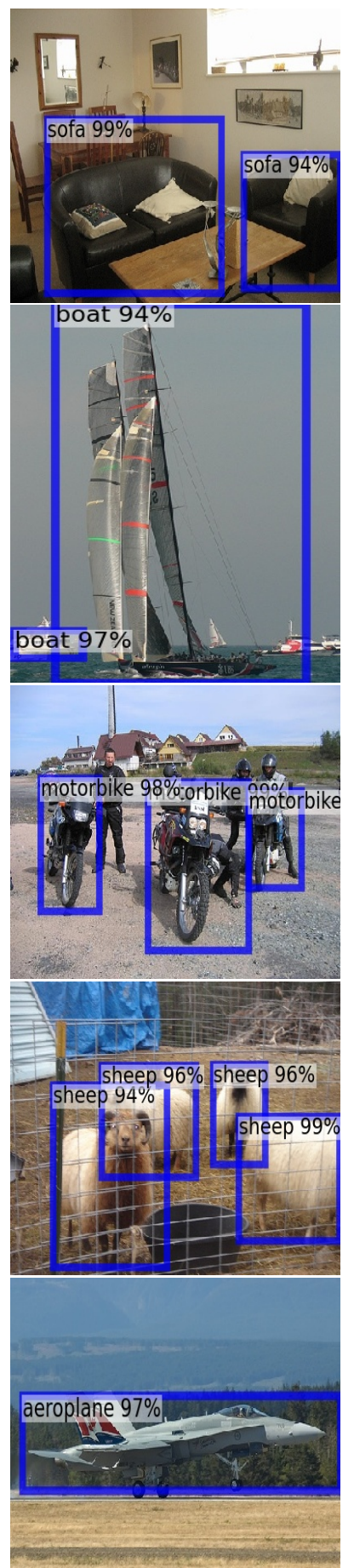

(d)

Figure A3: Qualitative Visualizations for the Ablation Study. (a) refers to the "weak" model, (b) refers to "weak $+\mathbf{S}_{c l s}^{l i n}+$ $\mathbf{S}_{c l s}^{v i s ", ~(c) ~ r e f e r s ~ t o ~ t h e ~ " w e a k ~}+\mathbf{S}_{c l s, r e g}^{l i n}+\mathbf{S}_{c l s, r e g}^{v i s}$ " with $k=0$, and (d) refers to the "weak $+\mathbf{S}_{c l s, r e g}^{l i n}+\mathbf{S}_{c l s, r e g}^{v i s}$ " after being trained on $k=5$ shots. Section $\mathrm{G}$ provides a detailed description of these models. 


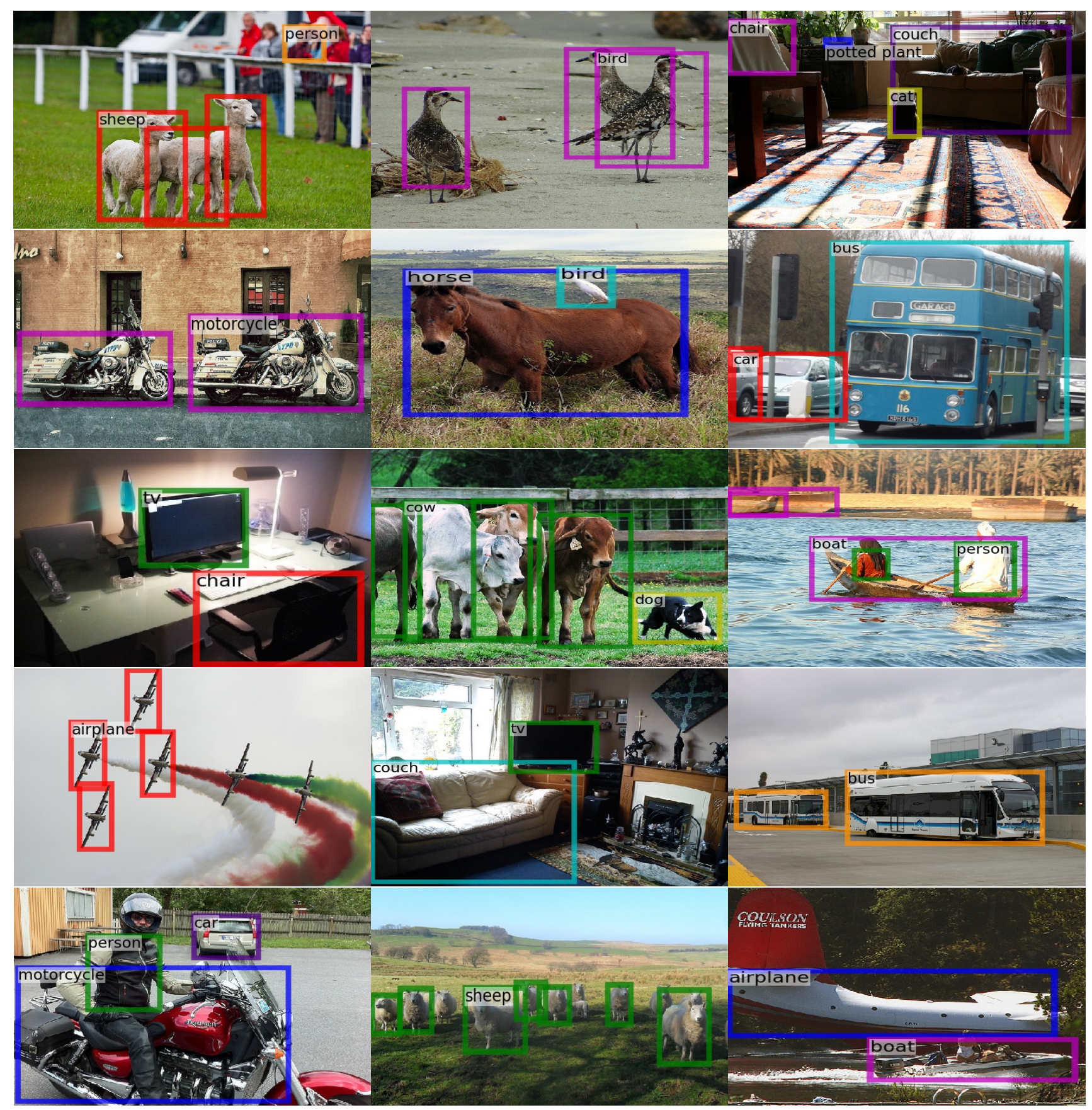

Figure A4: Qualitative Visualizations. Semi-supervised zero-shot $(k=0)$ detection performance on novel classes in MS-COCO (color = object category). 


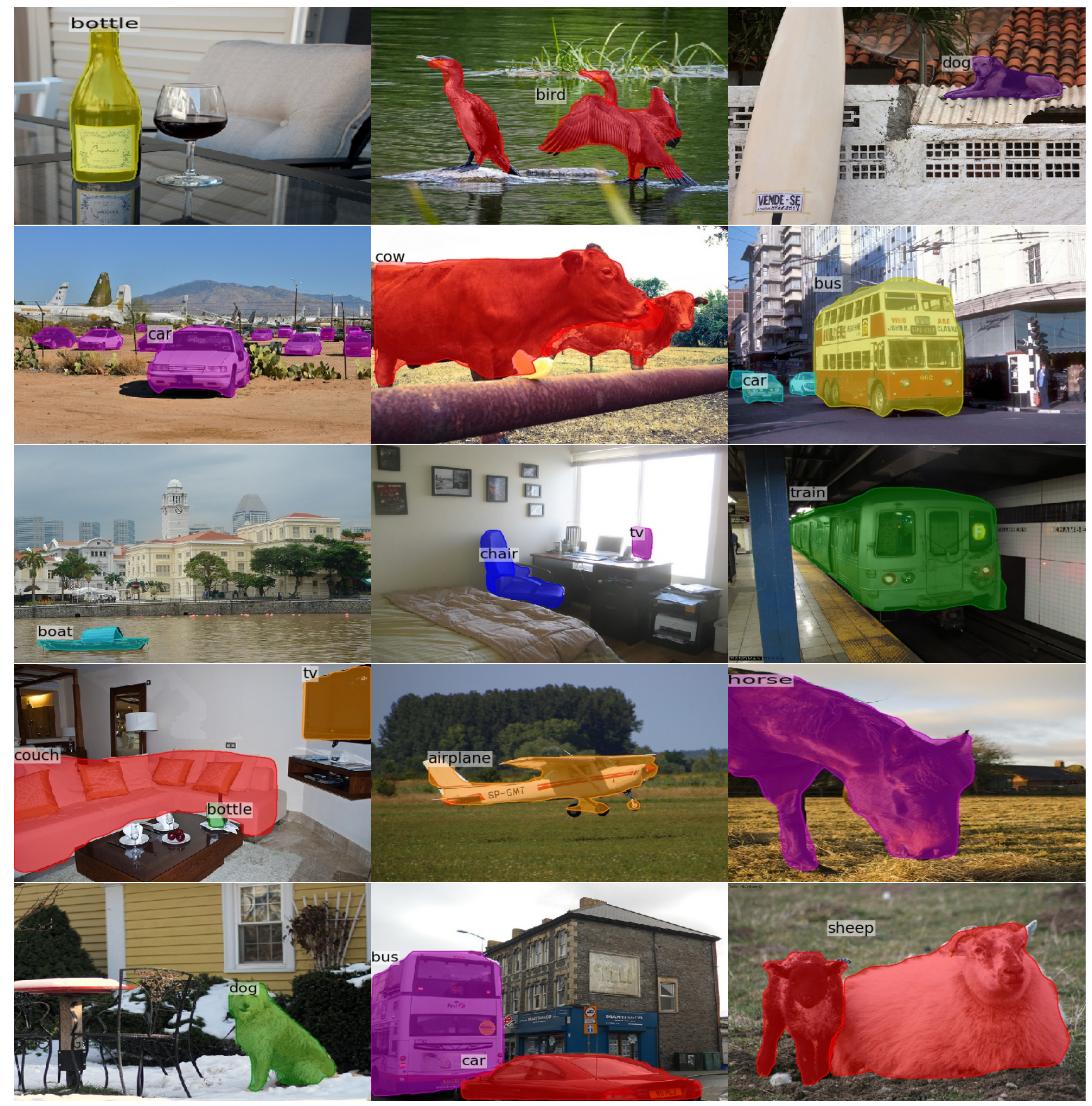

Figure A5: Qualitative Visualizations. Semi-supervised zero-shot $(k=0)$ instance segmentation performance on novel classes in MS-COCO (color $=$ object category). 